\title{
A flux-free a posteriori error estimator for the incompressible Stokes problem using a mixed FE formulation
}

\author{
Fredrik Larsson ${ }^{\mathrm{a}, *}$, Pedro Díez ${ }^{\mathrm{b}}$, Antonio Huerta $^{\mathrm{b}}$
}

\begin{abstract}
A B S T R A C T
In this contribution, we present an a posteriori error estimator for the incompressible Stokes problem valid for a conventional mixed FE formulation. Due to the saddle point property of the problem, conventional error estimators developed for pure minimization problems cannot be utilized straight forwardly. The new estimator is built up by two key ingredients. At first, a computed error approximation, exactly fulfilling the continuity equation for the error, is obtained via local Dirichlet problems. Secondly, we adopt the approach of solving local equilibrated flux free problems in order to bound the remaining, incompressible, error. In this manner, guaranteed upper and lower bounds, of the velocity "energy norm" of the error as well as goal oriented (linear) output functionals, with respect to a reference (overkill) mesh are obtained. In particular, it should be noted that this approach requires no computation of hybrid fluxes. Furthermore, the estimator is applicable to mixed FE formulations using continuous pressure approximations, such as the Mini and Taylor Hood class of elements. In conclusion, a few simple numerical examples are presented, illustrating the accuracy of the error bounds.
\end{abstract}

Keywords:

A posteriori error estimation Finite element method Incompressible Stokes flow Asymptotic bounds Flux-free error estimation

\section{Introduction}

The subject of error control in finite element analysis is of outmost importance in order to ascertain the accuracy of numerical computa tions. Since the seventies, global energy norm estimates have been developed for a large class of problems, see e.g. Babuška and Rheinboldt [1], Ainsworth and Oden [2] or Rüter and Stein [3]. More recently, error estimators in outputs of interest, so called Goal oriented error control, have been developed, cf. Eriksson et al. [4], Becker and Rannacher [5], Rannacher [6], Oden and Prudhomme [7] and Stein et al. [8]. For a quite comprehensive overview of error estimators, cf. Ainsworth and Oden [9] and Ladevèze and Pelle [10]. In the case of linear problems, recently developed estimators thrive at developing guaranteed upper and lower bounds for the outputs of interest, cf. Sauer Budge et al. [11], Parés et al. [12 16], Xuan et al. [17] and Ladevèze [18], while other approaches lead to asymptotic bounds, cf. Sauer Budge et al. [19] and Parés et al. [20]. The adjective asymptotic is used here meaning that the bounds are guaranteed with respect to a (much finer) reference mesh. For general non linear problems, these estimators usually aim at computing approximate bounds or approximations of the error itself, cf. Larsson et al. [21]. Developed estimators can advantageously be used in conjunction with adaptive procedures in order to optimize the computational mesh. Using goal oriented estimators for quantities of interest, the finite element mesh may thus be optimized to compute the desired output with a prescribed accuracy at lowest possible cost, cf. Díez and Calderón [22]. For a more in depth analysis on the behavior of adaptive procedures, cf. Dorfler [23], Morin et al. [24], Moon et al. [25] and Binev et al. [26].

The incompressible Stokes problem is an important archetype of a saddle point problem, appearing as a special case in creep flow, and some work has been devoted to the a posteriori error estimation for this problem. The finite element a priori error estimates and stability analysis for the problem are well studied in the literature, see e.g. Donea and Huerta [27]. Error estimates providing approximate bounds for the Stokes and Oseen equation were presented by Oden and Prudhomme [7] in terms of both the global energy type norm of the error and quantities of interest. Based on the method of variationally consistent post processing, estimators for outputs of interest were presented by Melbø and Kvamsdal [28]. In the works by Machiels et al. [29] and Paraschivoiu and Patera [30], asymptotic bounds were presented for outputs of interest. However, it should be noted that the derivations of these bounds were carried out for the Crouzeix Raviart elements, and heavily rely on the use of discontin uous pressure approximations. Furthermore, it should be noted that the estimators presented in $[29,30]$ require the solution of hybrid fluxes, equilibrating the element residuals. This procedure is cum bersome from the point of view of the implementation and requires 
extending and modifying conventional data structures. Furthermore, the solution of the hybrid fluxes is not unique, and must be chosen carefully to obtain accurate estimators.

In this paper, asymptotic bounds for outputs of interest of nu merical solution to the incompressible Stokes problem will be presented. An estimator that is, in contrast to existing (bounding) estimators, applicable also to finite element solutions with continuous pressure approximations will be derived based on local flux free problems. The use of so called flux free error estimators precludes the need for solving for the hybrid fluxes, otherwise needed to compute bounds for the error.

The remainder of the paper is outlined as follows: As point of departure, in Sections 2 and 3, we establish the error equation for the pertinent mixed FE approximation of the Stokes problem. In Section 4 we introduce a split of the error into two parts; a computable part which satisfies the error equation in terms of the incompressibility requirement, and a divergence free part, which can be controlled in terms of the velocity energy norm.

In Section 5 we describe the procedure for computing the first part of the error in terms of local Dirichlet problems. Depending on the nature of the (FE) pressure approximation, two alternatives are presented. In Section 5.1, local problems are formulated element wise for the case of discontinuous pressure approximations. In fact, this is much similar to the incompressible projection introduced by Machiels et al. [29]. For the case of continuous pressure approximations, the local Dirichlet problems are formulated patch wise on "stars" surrounding one node, $\mathrm{cf}$. Section 5.2.

Next, we target the remaining error equation for the divergence free part of the error as derived in Section 4. Due to the construction of the Dirichlet problems, the velocity energy norm of this part of the error can be shown to be bounded from above by a solution composed by solutions to local flux free problems formulated on "stars". The formulation of these problems is shown in Section 6 and is a straight forward extension of the work presented in [20].

In order to obtain also lower bounds for the velocity energy norm of the divergence free error, we may construct a second, uncon strained version of the local Dirichlet problems, cf. Section 5. The solution to these problems, subtracted by the previously mentioned computable part of the error, constructs an approximation of the divergence free error which is continuous and divergence free. We stress that these local problems are not necessary for bounding the error, but may be used to obtain sharper bounds.

The a posteriori error bound for the velocity energy norm of the total error is presented in Section 7, both by use of and without the solution of the unconstrained Dirichlet problems. The resulting estimators bound the velocity energy norm of the total error from above and below.

In Section 8, we introduce an error estimator for arbitrary linear output functionals of the solution. The estimator is obtained by fol lowing the ideas of, e.g. [4], and constructing a dual problem pertinent to the output functional describing a quantity of interest. By use of the bounding properties derived in the previous sections, we derive upper and lower bounds for the output functionals.

The paper is concluded in Section 9 by a few numerical examples illustrating the performance of the estimators.

\section{Problem formulation}

Consider the spatial domain $\Omega \subset \mathbb{R}^{d}$, with $d=2$ or 3 , with boundary $\Gamma=\overline{\Gamma_{N} \cup \Gamma_{D}}$. We now formulate the Stokes problem as

$-\boldsymbol{\sigma} \cdot \nabla=\boldsymbol{b}$ in $\Omega$

$\nabla \cdot \boldsymbol{u}=0 \quad$ in $\Omega$

$\boldsymbol{u}=\boldsymbol{u}_{D} \quad$ on $\Gamma_{\mathrm{D}}$ $\boldsymbol{\sigma} \cdot \boldsymbol{n}=\boldsymbol{t} \quad$ on $\Gamma_{\mathrm{N}}$

where $\boldsymbol{u}$ is the sought velocity field, $\boldsymbol{\sigma}$ is the Cauchy stress tensor, $\boldsymbol{b}$ is the given body force, and $\boldsymbol{u}_{\mathrm{D}}$ and $\boldsymbol{t}$ are prescribed velocity and traction, respectively, on the boundary. In order to close the set of equations, we introduce the constitutive relation for the Cauchy stress. Here we assume the linear (isotropic) Stokes' law,

$\boldsymbol{\sigma}=2 \mu(\boldsymbol{u} \otimes \nabla)^{\mathrm{sym}}-\boldsymbol{I} p$,

where $\mu$ is the viscosity coefficient and we introduced a new unknown in terms of the pressure $p$. The full problem in strong form thus reads to find the velocity $\boldsymbol{u}$ and pressure $p$ such that Eqs. (1) (5) hold.

The weak form of the problem reads as follows: Find $\boldsymbol{u}, p \in \mathcal{U} \times \mathcal{Q}$ such that

$$
\begin{aligned}
& a(\boldsymbol{u}, \boldsymbol{w})+b(p, \boldsymbol{w})=l_{\mathrm{u}}(\boldsymbol{w}) \quad \forall \boldsymbol{w} \in \mathcal{V}, \\
& b(q, \boldsymbol{u})=0 \quad \forall q \in \mathcal{Q},
\end{aligned}
$$

where we define the spaces

$$
\begin{aligned}
& \mathcal{U}=\left\{\boldsymbol{w} \in\left[H^{1}(\Omega)\right]^{d}: \boldsymbol{w}=\boldsymbol{u}_{D} \text { on } \Gamma_{\mathrm{D}}\right\}, \\
& \mathcal{V}=\left\{\boldsymbol{w} \in\left[H^{1}(\Omega)\right]^{d}: \boldsymbol{w}=\mathbf{0} \text { on } \Gamma_{\mathrm{D}}\right\}, \\
& \mathcal{Q}=\left\{q \in L_{2}(\Omega)\right\} .
\end{aligned}
$$

The introduced forms are defined as

$$
\begin{aligned}
& a(\boldsymbol{v}, \boldsymbol{w}):=\int_{\Omega}(\boldsymbol{w} \otimes \nabla)^{\mathrm{sym}}: 2 \mu(\boldsymbol{v} \otimes \nabla)^{\mathrm{sym}} d \Omega, \\
& b(q, \boldsymbol{w}):=-\int_{\Omega} q(\nabla \cdot \boldsymbol{w}) d \Omega, \\
& l_{\mathrm{u}}(\boldsymbol{w}):=\int_{\Omega} \boldsymbol{w} \cdot \boldsymbol{b} d \Omega+\int_{\Gamma_{\mathrm{N}}} \boldsymbol{w} \cdot \boldsymbol{t} d \Gamma,
\end{aligned}
$$$$
\text { for } \boldsymbol{v}, \boldsymbol{w} \in\left[H^{1}(\Omega)\right]^{d} \text { and } q \in L_{2}(\Omega) \text {. }
$$

Remark. For the case of solely prescribing the velocities on the boundaries, i.e. $\Gamma=\Gamma_{\mathrm{D}}$, adding a constant pressure will not affect the result and $\mathcal{Q}$ must be replaced by a reduced space $\mathcal{Q}^{\prime}$ in order for the problem to be solvable, i.e. in order for the pressure to be unique. For instance, the reduction can be introduced as

$\mathcal{Q}^{\prime}=\left\{q \in L_{2}(\Omega) ; \quad \int_{\Omega} q d \omega=0\right\}$.

Note that the velocity field is unique even in the case where the pressure is not.

\section{Finite element approximation and error equations}

First, we consider the (element) partitioning of the domain into NEL elements as

$\bar{\Omega}=\overline{U_{e} \Omega_{1} \Omega_{e}}$.

Furthermore, we shall introduce the notation $\partial \Omega_{e}$ for the boundary of a specific element and denote a single boundary $\Gamma_{k}$ where

$\overline{\bigcup_{e}^{N E L} \partial \Omega_{e}}=\overline{\bigcup_{k}^{N B} \Gamma_{k}}$

where $N B$ denotes the total number of boundaries. 
We shall now solve Eqs. (6) and (7) approximately by introducing discrete trial and test spaces for the velocity and pressure. It has been shown how to obtain bounds for output functionals to FE solutions computed using C R elements, cf. [29,30]. However, in this paper, we aim at presenting bounds for output functionals using a mixed variational FE discretization with continuous pressure approxima tions. The FE problem is thus stated as follows: Find $\boldsymbol{u}_{H}, p_{H} \in \mathcal{U}_{H} \times \mathcal{Q}_{H}$ such that

$a\left(\boldsymbol{u}_{H}, \boldsymbol{w}\right)+b\left(p_{H}, \boldsymbol{w}\right)=l_{u}(\boldsymbol{w}) \quad \forall \boldsymbol{w} \in \mathcal{V}_{H}$,

$b\left(q, \boldsymbol{u}_{H}\right)=0 \quad \forall q \in \mathcal{Q}_{H}$,

where $\mathcal{U}_{H} \subset \mathcal{U}, \mathcal{V}_{H} \subset \mathcal{V}$ and $\mathcal{Q}_{H} \subset \mathcal{Q}$ are the pertinent FE spaces. It is important to use the suitable combination of spaces for velocities and pressure such that the LBB condition is fulfilled in order to obtain a stabile solution.

We shall only consider continuous FE approximation of the velocities, whereby the trial and test space can be stated as follows:

$\mathcal{U}_{H}=\left\{\boldsymbol{w} \in\left[H^{1}(\Omega)\right]^{d}:\left.\boldsymbol{w}\right|_{\Omega_{k}} \in \mathcal{T}_{\mathrm{u}}\left(\Omega_{k}\right), \quad \boldsymbol{w}=\mathbf{u}_{\mathrm{D}}\right.$ on $\left.\Gamma_{\mathrm{D}}\right\}$,

$\mathcal{V}_{H}=\left\{\boldsymbol{w} \in\left[H^{1}(\Omega)\right]^{d}:\left.\boldsymbol{w}\right|_{\Omega_{e}} \in \mathcal{T}_{\mathrm{u}}\left(\Omega_{k}\right), \quad \boldsymbol{w}=0\right.$ on $\left.\Gamma_{\mathrm{D}}\right\}$

where $\mathcal{T}\left(\Omega_{k}\right)$ defines the approximation on element $k$. Turning to the pressure part, we shall consider two special classes of elements, those with (element wise) discontinuous pressure approximations and those with continuous pressure approximations. The finite element spaces can be stated as follows for a discretization using piecewise discontinuous pressure approximation:

$\mathcal{Q}_{H}=\hat{\mathcal{Q}}_{H}:=\left\{q \in L_{2}(\Omega):\left.q\right|_{\Omega_{k}} \in \mathcal{T}_{p}\left(\Omega_{k}\right)\right\}$,

where $\mathcal{T}_{\mathrm{p}}\left(\Omega_{k}\right)$ denotes the local approximation space for the pressure on element $k$. For the case of continuous pressure approximation, we replace the expression $\mathcal{Q}_{H}$ by

$\mathcal{Q}_{H}=\overline{\mathcal{Q}}_{H}:=\left\{q \in H^{1}(\Omega):\left.q\right|_{\Omega_{k}} \in \mathcal{T}_{p}\left(\Omega_{k}\right)\right\}$

In particular, we shall investigate 4 simple elements that satisfy the LBB condition, cf. [27]. The lowest order stable element with piecewise discontinuous pressure approximation is the P2P0 element, defined by

$\mathcal{T}_{u}(\Omega)=\left[\mathbb{P}_{2}(\Omega)\right]^{d}$,

$\mathcal{Q}_{H}=\hat{\mathcal{Q}}_{H}, \quad \mathcal{T}_{p}(\Omega)=\mathbb{P}_{0}(\Omega)$,

where $\mathbb{P}_{m}$ denotes the set of polynomials of degree $\leq m$. This element has linear convergence properties. An element with piecewise discontinuous pressure and quadratic convergence is the Crouzeix Raviart element defined by

$\mathcal{T}_{u}(\Omega)=\left[\mathbb{P}_{2}(\Omega)\right]^{d}+\mathbb{B}_{3}^{d}$,

$\mathcal{Q}_{H}=\hat{\mathcal{Q}}_{H}, \quad \mathcal{T}_{p}(\Omega)=\mathbb{P}_{1}(\Omega)$,

where $\mathbb{B}_{3}^{d}$ denotes the space of one cubic bubble enriching the polynomial approximation of the velocity. Turning to elements with continuous pressure approximation, we have the lowest order stable element, the Mini element, defined by

$\mathcal{T}_{u}(\Omega)=\left[\mathbb{P}_{1}(\Omega)\right]^{d}+\mathbb{B}_{3}^{d}$,
$\mathcal{Q}_{H}=\overline{\mathcal{Q}}_{H}, \quad \mathcal{T}_{p}(\Omega)=\mathbb{P}_{1}(\Omega)$,

which has linear convergence properties. Quadratic convergence is obtained for the Taylor Hood element, defined by

$\mathcal{T}_{u}(\Omega)=\left[\mathbb{P}_{2}(\Omega)\right]^{d}$,

$\mathcal{Q}_{H}=\overline{\mathcal{Q}}_{H}, \quad \mathcal{T}_{\mathrm{p}}(\Omega)=\mathbb{P}_{1}(\Omega)$,

which provides quadratic convergence properties.

Remark. Here, we have presented triangular/tetrahedral elements. The same quantities are obtained using the quadrilateral/hexagonal counterparts.

Defining the error for the finite element solution as

$\boldsymbol{e}:=\boldsymbol{u}-\boldsymbol{u}_{H}, \quad \boldsymbol{g}:=p-p_{H}$

we establish the error equation in terms of the problem of finding $(\boldsymbol{e}, g) \in \mathcal{V} \times \mathcal{Q}$ such that

$a(\boldsymbol{e}, \boldsymbol{w})+b(g, \boldsymbol{w})=R_{\mathrm{u}}(\boldsymbol{w}) \quad \forall \boldsymbol{w} \in \mathcal{V}$,

$b(q, \boldsymbol{e})=R_{\mathrm{p}}(q) \quad \forall q \in \mathcal{Q}$.

The residuals are defined as

$R_{u}(\boldsymbol{w}):=l_{u}(\boldsymbol{w})-a\left(u_{H}, \boldsymbol{w}\right)-b\left(p_{H}, \boldsymbol{w}\right)$

$R_{p}(q):=-b\left(q, \boldsymbol{u}_{H}\right)$

The important Galerkin orthogonality

$R_{u}(\boldsymbol{w})=a(\boldsymbol{e}, \boldsymbol{w})+b(g, \boldsymbol{w})=0 \quad \forall \boldsymbol{w} \in \mathcal{V}_{H}$,

$R_{p}(q)=b(q, \boldsymbol{e})=0 \quad \forall q \in \mathcal{Q}_{H}$,

follows the properties of the FE solution Eqs. (17) and (18).

\section{Divergence-split of the error}

We shall now proceed by bounding the error $(\boldsymbol{e}, g)$ using local solutions. In order to do that, we follow the idea by Machiels et al. [29] and introduce the intermediate solution $\left(\boldsymbol{u}_{\text {div }}, p_{\text {div }}\right)$ which is an approximation that fulfills the continuity equation exactly, i.e.

$b\left(q, u_{\text {div }}\right)=0 \quad \forall q \in \mathcal{Q}$.

Consequently, we introduce the split of the errors as

$\boldsymbol{e}=\boldsymbol{e}_{\mathrm{div}}+\boldsymbol{e}_{0}, \quad \boldsymbol{e}_{\mathrm{div}}:=\boldsymbol{u}_{\mathrm{div}}-\boldsymbol{u}_{\mathrm{H}}, \quad \boldsymbol{e}_{0}:=\boldsymbol{u}-\boldsymbol{u}_{\mathrm{div}}$,

$g=g_{\text {div }}+g_{0}, \quad g_{\text {div }}:=p_{\text {div }}-p_{H}, \quad g_{0}:=p-p_{\text {div }}$,

where $\bullet_{\text {div }}$ denotes the computable part of the error capturing the error of the divergence exactly and $\bullet_{0}$ denotes the divergence free error. For this split, we note that

$b\left(q, \boldsymbol{e}_{\mathrm{div}}\right)=R_{p}(q) \quad \forall q \in \mathcal{Q}$,

$b\left(q, \boldsymbol{e}_{0}\right)=0 \quad \forall q \in \mathcal{Q}$.

Indeed, as will be shown in the following sections, the divergence capturing error $\left(\boldsymbol{e}_{\mathrm{div}}, g_{\text {div }}\right)$ is computable. 
For the computed values of $\left(\boldsymbol{e}_{\mathrm{div}}, g_{\mathrm{div}}\right)$, we may define the divergence free residuals

$R_{u, \mathrm{div}}(\boldsymbol{w}):=R_{u}(\boldsymbol{w})-a\left(\boldsymbol{e}_{\mathrm{div}}, \boldsymbol{w}\right)-b\left(g_{\mathrm{div}}, \boldsymbol{w}\right)$,

$R_{p, \operatorname{div}}(q):=R_{p}(q)-b\left(q, \boldsymbol{e}_{\mathrm{div}}\right)=0$,

and the corresponding error equation for the remaining part as

$a\left(\boldsymbol{e}_{0}, w\right)+b\left(g_{0}, \boldsymbol{w}\right)=R_{u, \operatorname{div}}(\boldsymbol{w}) \quad \forall \boldsymbol{w} \in \mathcal{V}$

$b\left(q, \boldsymbol{e}_{0}\right)=0 \quad \forall q \in \mathcal{Q}$

where the solution $\left(\boldsymbol{e}_{0}, q_{0}\right) \in \mathcal{V} \times \mathcal{Q}$.

\section{Local Dirichlet problems for the divergence-capturing error}

We shall now construct the computable part of the error that equilibrates the divergence residual. Recall that we require that

$b\left(q, \boldsymbol{e}_{\mathrm{div}}\right)=R_{p}(q) \quad \forall q \in \mathcal{Q}$

holds exactly. There are infinitely many solutions $\boldsymbol{e}_{\mathrm{div}}$ that satisfy this requirement and thus, our split $\boldsymbol{e}=\boldsymbol{e}_{\mathrm{div}}+\boldsymbol{e}_{0}$ is not unique. Since the latter term is non computable, we wish for $\boldsymbol{e}_{\text {div }} \approx \boldsymbol{e}$ since this would result in a small $\boldsymbol{e}_{0}$, and thereby sharp bounds. However, we stress that the only requirement so far on $\boldsymbol{e}_{\mathrm{div}}$ is that stated in Eq. (47).

In order to construct the best possible approximation of the error, we shall start by computing localized approximations $\overline{\boldsymbol{e}}_{\mathrm{div}}, \bar{g}_{\mathrm{div}}$ that aim at being the best possible approximation of $(\boldsymbol{e}, \boldsymbol{g})$. The solution $\overline{\boldsymbol{e}}_{\text {div }}$ and the test space $\overline{\mathcal{V}}$ will be chosen such that the problem can be solved locally. Since the test space $\mathcal{Q}$ is infinite or at least much larger than $\mathcal{Q}_{H}$, this is an imperative feature. For the case of using the flux free error estimator, we further require that the lowest FE order Galerkin orthogonality holds for the updated residual,

$R_{u, \mathrm{div}}(\boldsymbol{w}):=R_{u}(\boldsymbol{w})-a\left(e_{\mathrm{div}}, \boldsymbol{w}\right)-b\left(g_{\mathrm{div}}, \boldsymbol{w}\right)=0 \quad \forall \boldsymbol{w} \in \mathcal{V}_{H, 1}$.

Here, the space $\mathcal{V}_{H, 1} \subset \mathcal{V}_{H}$ denotes the space of linear basis functions assumed to be part of the FE space, i.e.,

$\mathcal{V}_{H, 1}=\left\{\boldsymbol{w} \in \mathcal{V}_{H}:\left.\boldsymbol{w}\right|_{\Omega_{k}} \in\left[\mathbb{P}^{1}\left(\Omega_{k}\right)\right]^{d}\right\}$.

In the following, we present the procedure of computing $\left(\boldsymbol{e}_{\mathrm{div}}, g_{\mathrm{div}}\right)$ satisfying Eqs. (47) and (48) for the two important special cases of discontinuous and continuous pressure approximations in the FE formulation.

5.1. Computation of the divergence capturing error for discontinuous pressure approximations

Using elements with discontinuous pressure approximations, and thereby the element piecewise constant part of the test space, we ensure that the average divergence on an element is that of the prescribed one. Hence we can find a divergence controlled solution locally on $\Omega_{e}, \boldsymbol{u}_{\mathrm{div}}=\boldsymbol{u}_{H}+\boldsymbol{e}_{\mathrm{div}}$, where $\left.\boldsymbol{e}_{\mathrm{div}}\right|_{\Omega_{k}} \in\left[H^{1}\left(\Omega_{k}\right)\right]^{d}$ satisfies

$b_{k}\left(q, \boldsymbol{e}_{\mathrm{div}}\right)=R_{p, k}(q) \quad \forall q \in L_{2}\left(\Omega_{k}\right), \quad \boldsymbol{e}_{\mathrm{div}}=0 \quad$ on $\partial \Omega_{k}$.

where $b_{k}$ and $R_{p, k}$ denote the restriction of the forms to $\Omega_{k}$.

Following the ideas presented in [29], we adopt the apparent way of fulfilling this condition by solving local Dirichlet problems. On each element $k=1, \ldots, N E L$ we thus seek to find $\left(\overline{\boldsymbol{e}}_{\mathrm{div}, k}, \bar{g}_{\mathrm{div}, k}\right) \in \mathcal{V}_{0, k} \times \mathcal{Q}_{0, k}$ such that

$a_{k}\left(\bar{e}_{\mathrm{div}, k}, \delta \boldsymbol{w}\right)+b_{k}\left(\bar{g}_{\mathrm{div}, k}, \delta \boldsymbol{w}\right)=R_{u, k}(\delta \boldsymbol{w}) \quad \forall \delta \boldsymbol{w} \in \mathcal{V}_{0, k}$, $b_{k}\left(\delta q, \overline{\boldsymbol{e}}_{\mathrm{div}, k}\right)=R_{\mathrm{p}, k}(\delta q) \quad \forall \delta q \in \mathcal{Q}_{0, k}$,

where $a_{k}, b_{k}, R_{u, k}$ and $R_{p, k}$ denote the restriction of the forms to $\Omega_{k}$. Here, we introduced the local trial and test spaces

$\mathcal{V}_{0, k}:=\left\{w \in\left[H^{1}\left(\Omega_{k}\right)\right]^{d}: \boldsymbol{w}=0\right.$ on $\left.\partial \Omega_{k}\right\}$,

$\mathcal{Q}_{0, k}:=\left\{q \in L_{2}(\Omega): \int_{\Omega_{k}} q d \Omega=0\right\}$.

Note that here we have to restrict the trial and test spaces for the pressure in order for it to be unique since pure Dirichlet boundary conditions are prescribed for the velocities. This equals removing the piecewise constant from the test space. Hence, it is crucial that the boundary conditions are such that the conditions hold for a piecewise constant which, indeed, is the case when using discontinuous pressure approximation for $\left(\boldsymbol{u}_{H}, p_{H}\right)$. Since the piecewise constant on $\Omega_{k}$ is part of $\mathcal{Q}_{H}$, we have that

$0=-b\left(\left.1\right|_{\Omega_{k}}, \boldsymbol{u}_{H}\right)=R_{p}\left(\left.1\right|_{\Omega_{k}}\right)=R_{p, k}(1)=0$.

We now define the new solutions as the sum of the local solutions

$\overline{\boldsymbol{u}}_{\mathrm{div}}=\boldsymbol{u}_{H}+\overline{\boldsymbol{e}}_{\mathrm{div}},\left.\quad \overline{\boldsymbol{e}}_{\mathrm{div}}\right|_{\Omega_{k}}=\overline{\boldsymbol{e}}_{\mathrm{div}, k}$,

$\bar{p}_{\text {div }}=p_{H}+\bar{g}_{\text {div }},\left.\quad \bar{g}_{\text {div }}\right|_{\Omega_{k}}=\bar{g}_{\text {div }, k}$

which define an approximation that fulfills the equation of continuity Eq. (47) exactly.

For the case of using the flux free error estimator in Section 6, we require Galerkin orthogonality for the updated residual. In order to accommodate Eqs. (47) and (48), we formulate the expanded problem, of finding $\boldsymbol{e}_{\mathrm{div}, k}, g_{\mathrm{div}, k}, f_{k} \in \mathcal{V}_{0, k} \times \mathcal{Q}_{0, k} \times \overline{\mathcal{V}}_{H, 1, k}$ such that

$a_{k}\left(\boldsymbol{e}_{\mathrm{div}, k}, \boldsymbol{w}\right)+b_{k}\left(g_{\mathrm{div}, k}, \boldsymbol{w}\right)+a_{k}\left(f_{k}, \boldsymbol{w}\right)=R_{u, k}(\boldsymbol{w}) \quad \forall \boldsymbol{w} \in \mathcal{V}_{0, k}$,

$b_{k}\left(q, \boldsymbol{e}_{\mathrm{div}, k}\right)=R_{p, k}(q) \quad \forall q \in \mathcal{Q}_{0, k}$,

$a_{k}\left(\boldsymbol{e}_{\mathrm{div}, k}, \boldsymbol{w}_{H}\right)=0 \quad \forall \boldsymbol{w}_{H} \in \overline{\mathcal{V}}_{H, 1, k}$,

where we introduce quotient space resulting of suppressing the rigid body modes (the kernel of the bilinear form $a_{k}$ ) from the standard interpolation space $\mathcal{V}_{H, 1, k}$, namely

$\overline{\mathcal{V}}_{H, 1, k}:=\mathcal{V}_{H, 1, k} / \operatorname{kernel}\left(a_{k}\right)$

where the restriction of the (low order) FE space and the kernel of $a_{k}$ $(\bullet, \bullet)$ are defined as

$\mathcal{V}_{H, 1, k}:=\left\{\boldsymbol{w} \in\left[\mathbb{P}^{1}\left(\Omega_{k}\right)\right]^{d}: \boldsymbol{w}=0\right.$ on $\left.\partial \Omega_{k} \cap \Gamma_{\mathrm{D}}\right\}$,
$\operatorname{kernel}\left(a_{k}\right):=\left\{\boldsymbol{w} \in\left[H^{1}\left(\Omega_{k}\right)\right]^{d}: a_{k}(\boldsymbol{w}, \boldsymbol{w})=0\right\}$.

Note that, by definition of $\operatorname{kernel}\left(a_{k}\right)$,

$a_{k}\left(\boldsymbol{e}_{\mathrm{div}, k}, \boldsymbol{w}_{H}\right)=0 \quad \forall \boldsymbol{w}_{H} \in \operatorname{kernel}\left(a_{k}\right)$.

Furthermore, due to the restriction of the pressure space, we note that

$b(q, \boldsymbol{w})=-(\boldsymbol{w} \cdot \nabla) \int_{\Omega_{k}} q d \Omega=0$ 
for any $\boldsymbol{w} \in\left[\mathbb{P}^{1}\left(\Omega_{k}\right)\right]^{d} \subset \mathcal{V}_{H, 1, k}$ (with $\boldsymbol{\nabla} \cdot \boldsymbol{w}=$ constant) and $q \in \mathcal{Q}_{0, k}$. Hence, the solution to the problem above satisfies

$a_{k}\left(\boldsymbol{e}_{\mathrm{div}, k}, \boldsymbol{w}_{H}\right)+b_{k}\left(g_{\mathrm{div}, k}, \boldsymbol{w}_{H}\right)=0 \quad \forall \boldsymbol{w}_{H} \in \mathcal{V}_{H, 1, k}$.

Similarly as for the initial approximations, we now define the new solutions as the union of the local solutions

$\boldsymbol{u}_{\mathrm{div}}=\boldsymbol{u}_{H}+\boldsymbol{e}_{\mathrm{div}},\left.\quad \boldsymbol{e}_{\mathrm{div}}\right|_{\Omega_{k}}=\boldsymbol{e}_{\mathrm{div}, k}$,

$p_{\text {div }}=p_{H}+g_{\text {div }},\left.\quad g_{\text {div }}\right|_{\Omega_{k}}=g_{\text {div }, k}$.

which define an approximation that fulfills the equation of continuity Eq. (47) exactly. Clearly, since $\left.\boldsymbol{w}_{H}\right|_{\Omega_{k}} \in \mathcal{V}_{H, 1, k}$ for any $\boldsymbol{w}_{H} \in \mathcal{V}_{H, 1}$, we have that

$$
\begin{aligned}
R_{u, \mathrm{div}}(\boldsymbol{w}) & =R_{u}(\boldsymbol{w})-a\left(\boldsymbol{e}_{\mathrm{div}}, \boldsymbol{w}\right)-b\left(g_{\mathrm{div}}, \boldsymbol{w}\right) \\
& =R_{u}(\boldsymbol{w})-\sum_{k}^{N E L} 1 \underbrace{a\left(\boldsymbol{e}_{\mathrm{div}, k},\left.\boldsymbol{w}\right|_{\Omega_{k}}\right)+b\left(g_{\mathrm{div}, k},\left.\boldsymbol{w}\right|_{\Omega_{k}}\right)}_{0}] \\
& =R_{u}(\boldsymbol{w})=0 \quad \forall \boldsymbol{w} \in \mathcal{V}_{H, 1},
\end{aligned}
$$

whereby also Eq. (48) is enforced.

5.2. Computation of the divergence capturing error for continuous pressure approximations

We shall now present a strategy for computing a divergence exact velocity field in the case of using piecewise continuous approximation for the pressure. This is the case, for instance, using the conventional Mini or Taylor Hood class of mixed elements. The approach presented by $[29,30]$ can only be used in the case of discontinuous pressure approximation, cf. previous subsection, since the FE solution using continuous pressure approximation would not yield proper boundary conditions for local Dirichlet problems on element level.

We shall now proceed along the lines of [20], solving "flux free" problems on "stars" $\omega_{i}$, defined by the support for the linear, Lagrange, basis function $\Phi_{i}$ with a node in vertex $\boldsymbol{x}_{i}$. Using the partition of unity approach and homogeneous Dirichlet boundary conditions, we may define the solution spaces on each star as

$\mathcal{V}_{0}^{i}:=\left\{\boldsymbol{w} \in\left[H^{1}\left(\omega_{i}\right)\right]^{d}: \boldsymbol{w}=0\right.$ on $\left.\partial \omega_{i}\right\}$,

$\mathcal{Q}_{0}^{i}:=\left\{q \in L_{2}\left(\omega_{i}\right): \int_{\omega_{i}} q d \Omega=0\right\}$,

and solve the localized problems as that of finding $\left(\overline{\boldsymbol{e}}_{\text {div }}^{i}, \bar{g}_{\text {div }}^{i}\right) \in \mathcal{V}_{0}^{i} \times \mathcal{Q}_{0}^{i}$ such that

$a^{i}\left(\overline{\boldsymbol{e}}_{\mathrm{div}}^{i}, \boldsymbol{w}\right)+b^{i}\left(\bar{g}_{\mathrm{div}}^{i}, \boldsymbol{w}\right)=R_{\mathrm{u}}^{i}\left(\Phi_{i} \boldsymbol{w}\right) \quad \forall \boldsymbol{w} \in \mathcal{V}_{0}^{i}$

$b^{i}\left(q, \overline{\boldsymbol{e}}_{\mathrm{div}}^{i}\right)=R_{\mathrm{p}}^{i}\left(\Phi_{i} q\right) \quad \forall q \in \mathcal{Q}_{0}^{i}$,

where $a^{i}, b^{i}, R_{\mathrm{u}}^{i}$ and $R_{\mathrm{p}}^{i}$ denote the restriction of the forms to $\omega_{i}$. We now construct the global approximations as follows:

$\overline{\boldsymbol{u}}_{\mathrm{div}}=\boldsymbol{u}_{H}+\overline{\boldsymbol{e}}_{\mathrm{div}}, \quad \overline{\boldsymbol{e}}_{\mathrm{div}}=\sum_{i} \overline{\boldsymbol{e}}_{\mathrm{div}}^{i}$,

$\bar{p}_{\text {div }}=p_{H}+\bar{g}_{\text {div }}, \quad \bar{g}_{\text {div }}=\sum_{i} \bar{g}_{\text {div }}^{i}$
From its construction, it is clear that $\overline{\boldsymbol{e}}_{\text {div }}$ (and thereby $\left.\overline{\boldsymbol{u}}_{\text {div }}\right) \in \mathcal{V}$. Furthermore, $\boldsymbol{e}_{\text {div }}$ fulfills

$b\left(q, \overline{\boldsymbol{e}}_{\mathrm{div}}\right)=\sum_{i} b\left(q, \overline{\boldsymbol{e}}_{\mathrm{div}}^{i}\right)=\sum_{i} R_{\mathrm{p}}^{i}\left(\Phi_{i} q\right)=R_{p}(q) \quad \forall q \in \mathcal{Q}$

exactly. Since the constant $q$ is removed from $\mathcal{Q}^{i}$, it is required that $R_{p}^{i}$ $\left(\Phi_{i} 1\right)=0$. Indeed, this is ensured, since $R_{q, i}\left(\Phi_{i}\right)=0$ due to the Galerkin orthogonality when $\Phi_{i} \in \mathcal{Q}_{H}$.

Turning to the momentum equation, we note that $\overline{\boldsymbol{e}}_{\text {div }}$ and $\bar{g}_{\text {div }}$ do not fulfill Eq. (32) exactly since functions in $\overline{\mathcal{V}}^{i}$ cannot add up to any functions in $\mathcal{V}$. Similarly as for the case of piecewise constant pressure approximation, we now expand the system in order to accommodate for the constraint on the equilibrium pertinent to the flux free error estimator. Hence, we solve the expanded problem of finding $\boldsymbol{e}_{\text {div }}^{i}, \tilde{g}_{\text {div }}^{i}, f^{i} \in \mathcal{V}_{0}^{i} \times \mathcal{Q}_{0}^{i} \times \overline{\mathcal{V}}_{H, 1}^{i}$ such that

$a^{i}\left(\boldsymbol{e}_{\mathrm{div}}^{i}, \boldsymbol{w}\right)+b^{i}\left(\tilde{g}_{\mathrm{div}}^{i}, \boldsymbol{w}\right)+a^{i}\left(f^{i}, \boldsymbol{w}\right)=R_{u}^{i}\left(\Phi_{i} \boldsymbol{w}\right) \quad \forall \boldsymbol{w} \in \mathcal{V}_{0}^{i}$,

$b^{i}\left(q, \boldsymbol{e}_{\mathrm{div}}^{i}\right)=R_{p}^{i}\left(\Phi_{i} q\right) \quad \forall q \in \mathcal{Q}_{0}^{i}$,

$a^{i}\left(\boldsymbol{e}_{\mathrm{div}}^{i}, \boldsymbol{w}_{H}\right)=0 \quad \forall \boldsymbol{w}_{H} \in \overline{\mathcal{V}}_{H, 1}^{i}$,

where we introduced the space of linear FE interpolations with zero rigid body mode content, analogously to Eq. (61)

$\overline{\mathcal{V}}_{H, 1}^{i}:=\mathcal{V}_{H, 1}^{i} / \operatorname{kernel}\left(a^{i}\right)$

on star $i$. The restriction of the low order FE approximations and the kernel of $a^{i}(\cdot, \bullet)$ are defined as

$\nu_{H, 1}^{i}: \quad\left\{\boldsymbol{w} \in\left[H^{1}\left(\omega_{i}\right)\right]^{d}:\left.\boldsymbol{w}\right|_{\Omega_{k}} \in\left[\mathbb{P}^{1}\left(\Omega_{k}\right)\right]^{d} \forall k: \omega_{i} \cap \Omega_{k} \neq \varnothing, \quad \boldsymbol{w} \quad 0\right.$ on $\left.\partial \omega_{i} \cap \Gamma_{D}\right\}$,

$\operatorname{kernel}\left(a^{i}\right):=\left\{\boldsymbol{w} \in\left[H^{1}\left(\boldsymbol{\omega}_{i}\right)\right]^{d}: a^{i}(\boldsymbol{w}, \boldsymbol{w})=0\right\}$.

In order to ensure the lowest FE order Galerkin orthogonality Eq. (48), two alternatives of tackling the pressure term are found. One is to proceed by using Grahm Schmidt's orthogonalization method,

$g_{\text {div }}^{i}=\tilde{g}_{\text {div }}^{i}-\left.\sum_{\left.k\right|_{\Omega_{k} \cap \omega_{i} \neq \varnothing}} \frac{\left(\tilde{g}_{\text {div }}^{i}, 1\right)_{k}}{(1,1)_{k}} 1\right|_{\Omega_{k}}$,

which readily can be shown to yield

$b^{i}\left(g_{\text {div }}^{i}, \boldsymbol{w}\right)=0 \quad \forall \boldsymbol{w} \in \mathcal{V}_{H, 1}^{i}$,

since

$\boldsymbol{w} \in \mathcal{V}_{H, 1} \quad \Rightarrow(\nabla \cdot \boldsymbol{w}) \in\left\{q \in L_{2}(\Omega):\left.q\right|_{\Omega_{k}} \in \mathbb{P}^{0}\left(\Omega_{k}\right)\right\}$.

Alternatively, we set $g_{\text {div }}=0$ which fulfills Eq. (84) trivially.

Remark. The orthogonalization procedure Eq. (83) constructs a $g_{\text {div }}$ which is discontinuous. In the case of comparison with a reference mesh, i.e. $\mathcal{V}, \mathcal{Q} \approx \mathcal{V}_{h}, \mathcal{Q}_{h}$, this implies $g_{\text {div }} \notin \mathcal{Q}_{h}$. Setting $g_{\text {div }}=0$ circumvents the problem.

Constructing the global approximations,

$\boldsymbol{u}_{\mathrm{div}}=\boldsymbol{u}_{H}+\boldsymbol{e}_{\mathrm{div}}, \quad \boldsymbol{e}_{\mathrm{div}}=\sum_{i} \boldsymbol{e}_{\mathrm{div}}^{i}$, 
$p_{\text {div }}=p_{H}+g_{\text {div }}, \quad g_{\text {div }}=\sum_{i} g_{\text {div }}^{i}$

we see that, as for the case of the approximations $\left(\overline{\boldsymbol{e}}_{\mathrm{div}}^{i}, \bar{g}_{\mathrm{div}}^{i}\right), \boldsymbol{e}_{\mathrm{div}}$ fulfills the continuity condition Eq. (47) exactly. Furthermore, since $\left.\mathcal{V}_{H, 1} \ni \boldsymbol{w}_{H}\right|_{\omega_{i}} \in \mathcal{V}_{H, 1}^{i}$, we have that

$$
\begin{aligned}
R_{u, \mathrm{div}}(\boldsymbol{w}) & =R_{u}(\boldsymbol{w})-a\left(\boldsymbol{e}_{\mathrm{div}}, \boldsymbol{w}\right)-b\left(g_{\mathrm{div}}, \boldsymbol{w}\right) \\
& =R_{u}(\boldsymbol{w})-\sum_{i}[\underbrace{a^{i}\left(\boldsymbol{e}_{\mathrm{div}}^{i},\left.\boldsymbol{w}\right|_{\omega_{i}}\right)+b^{i}\left(g_{\mathrm{div}}^{i},\left.\boldsymbol{w}\right|_{\omega_{i}}\right)}_{0}] \\
& =R_{u}(\boldsymbol{w})=0 \quad \forall \boldsymbol{w} \in V_{H, 1},
\end{aligned}
$$

whereby also Eq. (48) is enforced.

\section{A flux-free error estimator}

\subsection{Computation of a broken solution of the divergence free error}

Turning to the error $\left(\boldsymbol{e}_{0}, g_{0}\right)$, in order to compute subsequent error bounds, we first introduced the broken space, relaxing the continuity of the velocities,

$\hat{\mathcal{V}}:=\left\{\boldsymbol{w} \in\left[\hat{H}_{H}^{1}(\Omega)\right]^{d}: \boldsymbol{w}=\mathbf{0}\right.$ on $\left.\Gamma_{\mathrm{D}}\right\}$,

where the broken Sobolev space is defined as

$\hat{H}_{H}^{1}(\Omega):=\left\{\boldsymbol{w} \in L_{2}(\Omega):\left.\boldsymbol{w}\right|_{\Omega_{e}} \in H^{1}\left(\Omega_{e}\right)\right\}$

Hence, we note that $\left[H^{1}(\Omega)\right]^{d}, \mathcal{U}, \mathcal{V} \subset\left[\hat{H}_{H}^{1}(\Omega)\right]^{d}$ and, in particular, $\mathcal{V} \subset \hat{\mathcal{V}}$.

In order to be consequent, we redefine our forms $a, b$ and $l_{u}$ in order to act on the broken space,

$a:\left[\hat{H}_{H}^{1}(\Omega)\right]^{d} \times\left[\hat{H}_{H}^{1}(\Omega)\right]^{d} \rightarrow \mathbb{R}: \quad a(\boldsymbol{v}, \boldsymbol{w}):=\sum_{k}^{N E L} a_{k}\left(\left.\boldsymbol{v}\right|_{\Omega_{k}},\left.\boldsymbol{w}\right|_{\Omega_{k}}\right)$,

$b: L_{2}(\Omega) \times\left[\hat{H}_{H}^{1}(\Omega)\right]^{d} \rightarrow \mathbb{R}: \quad b(q, \boldsymbol{w}):=\sum_{k}^{N E L} b_{k}\left(\left.q\right|_{\Omega_{k}},\left.\boldsymbol{w}\right|_{\Omega_{k}}\right)$,

$l_{u}:\left[\hat{H}_{H}^{1}(\Omega)\right]^{d} \rightarrow \mathbb{R}: \quad l_{u}(\boldsymbol{w}):=\sum_{k}^{N E L} l_{\mathrm{u}, k}\left(\left.\boldsymbol{w}\right|_{\Omega_{k}}\right)$

where

$a_{k}(\boldsymbol{v}, \boldsymbol{w}):=\int_{\Omega_{k}}(w \otimes \nabla)^{\mathrm{sym}}: 2 \mu(\boldsymbol{v} \otimes \nabla)^{\mathrm{sym}} d \Omega$,

$b_{k}(q, \boldsymbol{w}):=-\int_{\Omega_{k}} q(\nabla \cdot \boldsymbol{w}) d \Omega$,

$l_{u, k}(\boldsymbol{w}):=\int_{\Omega_{k}} \boldsymbol{w} \cdot b d \Omega+\int_{\Gamma_{\mathrm{N}} \cap \partial \Omega_{k}} \boldsymbol{w} \cdot t d \Gamma$.

Note that for any functions $\boldsymbol{w}, \boldsymbol{v} \in \mathcal{U} \times \mathcal{V}$ and $q \in \mathcal{Q}$, these definitions are identical to the previous ones.

Following the ideas in [20], we now proceed by using the partition of unity approach. We assume that each vertex $\boldsymbol{x}_{i}$ in the mesh is related to a (linear) shape function $\Phi_{i}(\boldsymbol{x})$ and a "star" $\omega_{i}=\sup \Phi_{i}$ Choosing the conventional Lagrange shape functions, we note that $\sum_{i} \Phi_{i}=1$.

We are now in the position to state the local problems as follows: For each star $i$, find $\left(\hat{\boldsymbol{e}}^{i}, \hat{g}^{i}\right) \in \overline{\mathcal{V}}^{i} \times \mathcal{Q}^{i}$ such that

$a^{i}\left(\hat{\boldsymbol{e}}^{i}, \boldsymbol{w}\right)+b^{i}\left(\hat{g}^{i}, \boldsymbol{w}\right)=R_{u, \operatorname{div}}^{i}\left(\Phi_{i}\left(\boldsymbol{w}-\pi_{H, 1} \boldsymbol{w}\right)\right) \quad \forall \boldsymbol{w} \in \overline{\mathcal{V}}^{i}$, $b^{i}\left(q, \hat{\boldsymbol{e}}^{i}\right)=0 \quad \forall q \in \mathcal{Q}^{i}$,

where $a^{i}, b^{i}$ and $R_{u, \mathrm{div}}^{i}$ denote the restriction of the forms to $\omega_{i}$. The trial and test spaces for the broken problems are the following:

$\overline{\mathcal{V}}^{i}:=\mathcal{V}^{i} / \operatorname{kernel}\left(a^{i}\right)$

$\mathcal{Q}^{i}:=L_{2}\left(\omega_{i}\right)$.

where the restriction of the test space and the kernel of $a^{i}(\cdot, \cdot)$ are defined as

$\mathcal{V}^{i}:=\left\{\boldsymbol{w} \in\left[H^{1}\left(\omega_{i}\right)\right]^{d} \mid \boldsymbol{w}=\mathbf{0}\right.$ on $\left.\partial \omega_{i} \cap \Gamma_{\mathrm{D}}\right\}$,

$\operatorname{kernel}\left(a^{i}\right):=\left\{\boldsymbol{w} \in\left[H^{1}\left(\boldsymbol{\omega}_{i}\right)\right]^{d}: a^{i}(\boldsymbol{w}, \boldsymbol{w})=0\right\}$

The operator $\pi_{H, 1}$ denotes the (nodal) interpolation onto the $\mathrm{FE}$ space of piecewise linear velocity approximation $\mathcal{V}_{H, 1}$. Clearly, since any function $w \in \mathcal{V}^{i} \backslash \overline{\mathcal{V}}^{i}$ lies in $\mathcal{V}^{i} \cap \operatorname{kernel}\left(a^{i}\right) \subset \mathcal{V}_{H, 1}^{i}$ given in Eq. (81), which embodies all linear velocity fields on $\omega_{i}, \boldsymbol{w}-\pi_{H, 1} \boldsymbol{w} \equiv \mathbf{0}$. Hence, Eq. (97) holds for any restriction $\left.\boldsymbol{w}\right|_{\Omega_{k}} \in \mathcal{V}^{i}$ of any test function $\boldsymbol{w} \in \mathcal{V}$.

We now state the global solution as

$\hat{\boldsymbol{e}}:=\sum_{i} \hat{\boldsymbol{e}}^{i}, \quad \hat{g}=\sum_{i} \hat{g}^{i}$

that satisfies the error equation,

$$
\begin{aligned}
a(\hat{\boldsymbol{e}}, \boldsymbol{w})+b(\hat{g}, \boldsymbol{w}) & =\sum_{i} a^{i}\left(\hat{\boldsymbol{e}}^{i}, \boldsymbol{w}\right)+b^{i}\left(\hat{g}^{i}, \boldsymbol{w}\right) \\
& =\sum_{i} R_{u, \operatorname{div}}^{i}\left(\Phi_{i}\left(\boldsymbol{w}-\pi_{H, 1} \boldsymbol{w}\right)\right) \\
& =R_{u, \operatorname{div}}\left(\boldsymbol{w}-\pi_{H, 1} \boldsymbol{w}\right)=R_{u, \operatorname{div}}(\boldsymbol{w}) \quad \forall \boldsymbol{w} \in V,
\end{aligned}
$$

due to the Galerkin orthogonality in $R_{\mathrm{u}, \mathrm{div}}(\bullet)$, and for which

$b(q, \hat{\boldsymbol{e}})=\sum_{i} b^{i}\left(q, \hat{\boldsymbol{e}}^{i}\right)=0 \quad \forall q \in \mathcal{Q}$.

Remark. Note that we here use the fact the Galerkin orthogonality holds for $R_{u, \text { div }}$. Hence, it is crucial that we use the fully equilibrated local solution $\boldsymbol{e}_{\text {div }}$.

\subsection{Estimation of the error in the velocity energy norm}

We shall now consider the velocity energy norm of the error $\|\boldsymbol{e}\|$, where

$\|\boldsymbol{w}\|:=\sqrt{ } a(\boldsymbol{w}, \boldsymbol{w})$.

Note that for the space $\{\boldsymbol{w} \in \mathcal{V}: b(q, \boldsymbol{w})=0 \forall q \in \mathcal{Q}\}$, $\|\boldsymbol{e}\|$ is the energy norm of the problem. Furthermore, we note that $\|\bullet\|$ constitutes a norm on $\mathcal{V}$. 
Since both ê and $\boldsymbol{e}_{0}$ are divergence free and satisfy the error equation, we may bound the difference between the representations of the error as follows:

$$
\begin{aligned}
\left\|\boldsymbol{e}_{0}-\hat{\boldsymbol{e}}\right\|^{2} & =\left\|\boldsymbol{e}_{0}\right\|^{2}+\|\hat{\boldsymbol{e}}\|^{2}-2 a\left(\boldsymbol{e}_{0}, \hat{\boldsymbol{e}}\right) \\
& =\left\|\boldsymbol{e}_{0}\right\|^{2}+\|\hat{\boldsymbol{e}}\|^{2}-2[R_{u, \operatorname{div}}\left(\boldsymbol{e}_{0}\right)-\underbrace{b\left(\hat{g}, \boldsymbol{e}_{0}\right)}_{0}] \\
& =\left\|\boldsymbol{e}_{0}\right\|^{2}+\|\hat{\boldsymbol{e}}\|^{2}-2[a\left(\boldsymbol{e}_{0}, \boldsymbol{e}_{0}\right)+\underbrace{b\left(g_{0}, \boldsymbol{e}_{0}\right)}_{0}] \\
& =\|\hat{\boldsymbol{e}}\|^{2}-\left\|\boldsymbol{e}_{0}\right\|^{2},
\end{aligned}
$$

whereby

$\left\|\boldsymbol{e}_{0}\right\| \leq\|\hat{\boldsymbol{e}}\|=:\left\|\boldsymbol{e}_{0}\right\|_{\mathrm{UB}}$.

Furthermore, in order to bound $\left\|\boldsymbol{e}_{0}\right\|$ from below, we shall consider the proper minimization problem pertinent to Eqs. (45) and (46), as follows:

$$
\begin{aligned}
\left\|\boldsymbol{e}_{0}\right\|^{2}=a\left(\boldsymbol{e}_{0}, \boldsymbol{e}_{0}\right)= & -2 \inf _{\boldsymbol{w} \in \mathcal{V}} \frac{1}{2} a(\boldsymbol{w}, \boldsymbol{w})-R_{u, \operatorname{div}}(\boldsymbol{w}) \\
& \text { s.t. } \quad b(q, \boldsymbol{w})=0 \quad \forall q \in \mathcal{Q} \\
= & \sup _{\boldsymbol{w} \in \mathcal{V}} 2 R_{u, \operatorname{div}}(\boldsymbol{w})-a(\boldsymbol{w}, \boldsymbol{w}) \\
& \text { s.t. } \quad b(q, \boldsymbol{w})=0 \quad \forall q \in \mathcal{Q} \\
\geq & 2 R_{u, \operatorname{div}}(\varphi)-a(\varphi, \varphi),
\end{aligned}
$$

for any $\varphi \in \mathcal{V}$ that is divergence free,

$$
b(q, \varphi)=0 \quad \forall q \in \mathcal{Q} .
$$

Note that, due to the equilibration of $\boldsymbol{e}_{\mathrm{div}}$, we expect $\overline{\boldsymbol{e}}_{\mathrm{div}}$ to be a better approximation of the error than $\boldsymbol{e}_{\text {div }}$. Hence, we may use the lower bound estimate

$\boldsymbol{\varphi}=\overline{\boldsymbol{e}}_{\mathrm{div}}-\boldsymbol{e}_{\mathrm{div}}=: \overline{\boldsymbol{e}}$

which is in $\mathcal{V}$ and is divergence free, since

$b(q, \overline{\boldsymbol{e}})=b\left(q, \overline{\boldsymbol{e}}_{\mathrm{div}}\right)-b\left(q, \boldsymbol{e}_{\mathrm{div}}\right)=R_{p}(q)-R_{p}(q)=0 \quad \forall q \in \mathcal{Q}$.

Furthermore, since $\mathcal{v}$ is a linear space and

$b(q, \alpha \overline{\boldsymbol{e}})=\alpha b(q, \overline{\boldsymbol{e}})=0 \quad \forall q, \alpha \in \mathcal{Q} \times \mathbb{R}$,

we may chose any $\varphi=\alpha \overline{\boldsymbol{e}}$ as the argument. Hence, we may define the lower bound by

$\left\|\boldsymbol{e}_{0}\right\|^{2} \geq\left\|\boldsymbol{e}_{0}\right\|_{L B}^{2}$

where

$$
\begin{aligned}
\left\|\boldsymbol{e}_{0}\right\|_{L B}^{2} & =\max _{\alpha} 2 R_{u, \operatorname{div}}(\alpha \overline{\boldsymbol{e}})-\|\alpha \overline{\boldsymbol{e}}\|^{2} \\
& =\max _{\alpha} 2 R_{u, \operatorname{div}}(\overline{\boldsymbol{e}}) \alpha-\|\overline{\boldsymbol{e}}\|^{2} \alpha^{2} \\
& =\frac{\left(R_{u, \operatorname{div}}(\overline{\boldsymbol{e}})\right)^{2}}{\|\overline{\boldsymbol{e}}\|^{2}},
\end{aligned}
$$

for any computed solution $\overline{\mathbf{e}} \neq \mathbf{0}$. As an alternative, directly inserting $\boldsymbol{\varphi}=\mathbf{0}$ as the argument, we obtain the result, which is also apparent from the norm property of $\|\bullet\|$. Without computing an additional local solution, this yields a lower bound.
Remark. For the case that we use discontinuous pressure approxi mation in $\mathcal{Q}_{H}$, the dimension of the maximization problem is increased to that of the number of elements in $\mathcal{V}_{H}$ since

$\varphi=\sum_{k}^{N E L} \alpha_{k} \overline{\boldsymbol{e}}_{k}=\sum_{k}^{N E L} \alpha_{k}\left(\overline{\boldsymbol{e}}_{\mathrm{div}, k}-\boldsymbol{e}_{\mathrm{div}, k}\right)$

are admissible candidates $\in \mathcal{V}$ satisfying Eq. (110). This larger maxi mization problem results in the sharper bound

$\left\|\boldsymbol{e}_{0}\right\|_{L B}^{2}=\sum_{k}^{N E L} \frac{\left(R_{u, \operatorname{div}, k}(\overline{\boldsymbol{e}})\right)^{2}}{\left\|\overline{\boldsymbol{e}}_{k}\right\| \Omega_{k} 2}$.

In summary, we have presented bounds for $\boldsymbol{e}_{0}$ that can be stated as

$\left\|\boldsymbol{e}_{0}\right\|_{L B}^{2} \leq\left\|\boldsymbol{e}_{0}\right\|^{2} \leq\left\|\boldsymbol{e}_{0}\right\|_{U B}^{2}$.

\section{Bounding the error using the triangle inequality}

In the previous section, upper and lower bounds for the velocity energy norm of the divergence free error were presented. We conclude that $\|\hat{\mathbf{e}}\|$ is simply the upper bound of the energy norm error of the improved solution $\boldsymbol{u}_{\mathrm{div}}, p_{\mathrm{div}}$. In this section, we shall derive bounds for the velocity energy norm of the total error for the finite element solution. Such bounds can be obtained using the triangle inequality together with the fact that one part of the error, the divergence capturing part $\boldsymbol{e}_{\mathrm{div}}$, is computed. The straight forward, naive, application gives the bounds

$\left\|\boldsymbol{e}_{\mathrm{div}}\right\|-\left\|\boldsymbol{e}_{0}\right\|_{U B} \leq\|\boldsymbol{e}\| \leq\left\|\boldsymbol{e}_{\mathrm{div}}\right\|+\left\|\boldsymbol{e}_{0}\right\|_{U B}$.

However, it turns out that these bounds can be tightened using further computed solutions. Below, we present bounds optimized in terms of minimum bound gap.

\subsection{Optimized triangle inequality bounds, without improved solution}

In order to improve the triangle inequality above, we shall use the triangle inequality after adding and subtracting an arbitrary constant times the broken solution $\hat{\mathbf{e}}$ computed in order to establish the upper bound of $\left\|\boldsymbol{e}_{0}\right\|$, i.e.

$\|\boldsymbol{e}\|=\left\|\boldsymbol{e}_{\mathrm{div}}+e_{0}\right\|=\left\|\boldsymbol{e}_{\mathrm{div}}+\alpha \hat{\boldsymbol{e}}+\boldsymbol{e}_{0}-\alpha \hat{\boldsymbol{e}}\right\|$

Hence, we may construct the generalized triangle inequality bounds as

$\left\|\boldsymbol{e}_{\mathrm{div}}+\alpha \hat{\boldsymbol{e}}\right\|-\left\|\boldsymbol{e}_{0}-\alpha \hat{\boldsymbol{e}}\right\| \leq\|\boldsymbol{e}\| \leq\left\|\boldsymbol{e}_{\mathrm{div}}+\alpha \hat{\boldsymbol{e}}\right\|-\left\|\boldsymbol{e}_{0}-\alpha \hat{\boldsymbol{e}}\right\|$.

In a generic format, we state the following

$\bar{E}-\frac{\Delta E}{2} \leq\|\boldsymbol{e}\| \leq \bar{E}+\frac{\Delta E}{2}$,

where

$\bar{E}=\left\|\boldsymbol{e}_{\mathrm{div}}+\alpha \hat{\boldsymbol{e}}\right\|$,

and we shall construct $\Delta E$ such that

$2\left\|\boldsymbol{e}_{0}-\alpha \hat{\boldsymbol{e}}\right\| \leq \Delta E$.

To this end, we expand the square of the term to be bounded as follows:

$\left\|\boldsymbol{e}_{0}-\alpha \hat{\boldsymbol{e}}\right\|^{2}=(1-2 \alpha)\left\|\boldsymbol{e}_{0}\right\|^{2}+\alpha^{2}\|\hat{\boldsymbol{e}}\|^{2}$. 
Depending on the sign of $(1-2 \alpha)$, three different bounds are given,

$\left\|\boldsymbol{e}_{0}-\alpha \hat{\boldsymbol{e}}-\beta \overline{\boldsymbol{e}}\right\| 2 \leq\left(\frac{\Delta E}{2}\right)^{2}= \begin{cases}(1-\alpha)^{2}\|\hat{\boldsymbol{e}}\|^{2} & \text { if } \alpha<\frac{1}{2} \\ \frac{1}{4}\|\hat{\boldsymbol{e}}\|^{2} & \text { if } \alpha=\frac{1}{2} \\ \alpha^{2}\|\hat{\boldsymbol{e}}\|^{2} & \text { if } \alpha>\frac{1}{2},\end{cases}$

where we made use of the inequalities

$0 \leq\left\|\boldsymbol{e}_{0}\right\|^{2} \leq\|\hat{\boldsymbol{e}}\|^{2}$

Evaluating the variation of these quadratic bounds gives us the optimal choice of $\alpha$ that minimizes $\Delta E$. There are no stationary points for $\alpha<1 / 2$ or $\alpha>1 / 2$. Hence, the optimal value is $\alpha=1 / 2$. Hence we may conclude the optimal bounds as follows:

$\left\|\boldsymbol{e}_{\mathrm{div}}+\frac{1}{2} \hat{\boldsymbol{e}}\right\|-\frac{1}{2} \hat{\boldsymbol{e}}\|\leq\| \boldsymbol{e}\|\leq\| \boldsymbol{e}_{\mathrm{div}}+\frac{1}{2} \hat{\boldsymbol{e}}\left\|+\frac{1}{2}\right\| \hat{\boldsymbol{e}} \|$

which clearly narrows the bound gap in comparison to the straight forward use of the triangle inequality.

\subsection{Optimized triangle inequality bounds, with improved solution}

Generalizing the rough strategy in the previous subsection, we may utilize the information obtained in terms of the solutions $\hat{\mathbf{e}}$ and $\overline{\mathbf{e}}$ computed in order to assess upper and lower bounds for $\left\|\boldsymbol{e}_{0}\right\|$, respectively. Doing so, we add and subtract these solutions inside the norm expression as follows:

$\|\boldsymbol{e}\|=\left\|\boldsymbol{e}_{\mathrm{div}}+\boldsymbol{e}_{0}\right\|=\left\|\boldsymbol{e}_{\mathrm{div}}+\alpha \hat{\boldsymbol{e}}+\beta \overline{\boldsymbol{e}}+\boldsymbol{e}_{0}-\alpha \hat{\boldsymbol{e}}-\beta \overline{\boldsymbol{e}}\right\|$,

where $\alpha$ and $\beta$ are arbitrary constants that we later shall optimize in order to sharpen the bound. The triangle inequality may now be used to compute establish the upper bound

$\|\boldsymbol{e}\| \leq\left\|\boldsymbol{e}_{\mathrm{div}}+\alpha \hat{\boldsymbol{e}}+\beta \overline{\boldsymbol{e}}\right\|+\left\|\boldsymbol{e}_{0}-\alpha \hat{\boldsymbol{e}}-\beta \overline{\boldsymbol{e}}\right\|$

and the lower bound

$\|\boldsymbol{e}\| \geq\left\|\boldsymbol{e}_{\mathrm{div}}+\alpha \hat{\boldsymbol{e}}+\beta \overline{\boldsymbol{e}}\right\|-\left\|\boldsymbol{e}_{0}-\alpha \hat{\boldsymbol{e}}-\beta \overline{\boldsymbol{e}}\right\|$.

In a generic format, we state the following

$\bar{E}-\frac{\Delta E}{2} \leq\|\boldsymbol{e}\| \leq \bar{E}+\frac{\Delta E}{2}$,

where

$\bar{E}=\left\|\boldsymbol{e}_{\mathrm{div}}+\alpha \hat{\boldsymbol{e}}+\beta \overline{\boldsymbol{e}}\right\|$,

and we shall construct $\Delta E$ such that

$2\left\|\boldsymbol{e}_{0}-\alpha \hat{\boldsymbol{e}}-\beta \overline{\boldsymbol{e}}\right\| \leq \Delta E$

To this end, we expand the square of the term to be bounded as follows:

$\left\|\boldsymbol{e}_{0}-\alpha \hat{\boldsymbol{e}}-\beta \overline{\boldsymbol{e}}\right\|^{2}=(1-2 \alpha)\left\|\boldsymbol{e}_{0}\right\|^{2}+\alpha^{2}\|\hat{\boldsymbol{e}}\|^{2}+2(\alpha-1) \beta a(\hat{\boldsymbol{e}}, \overline{\boldsymbol{e}})+\beta^{2}\|\overline{\boldsymbol{e}}\|^{2}$
Depending on the sign of $(1-2 \alpha)$, three different bounds are given,

$$
\begin{aligned}
& \left\|\begin{array}{lll}
\| \boldsymbol{e}_{0} & \alpha \hat{\boldsymbol{e}} & \beta \overline{\boldsymbol{e}}
\end{array}\right\|^{2} \leq\left(\frac{\Delta E}{2}\right)^{2}
\end{aligned}
$$

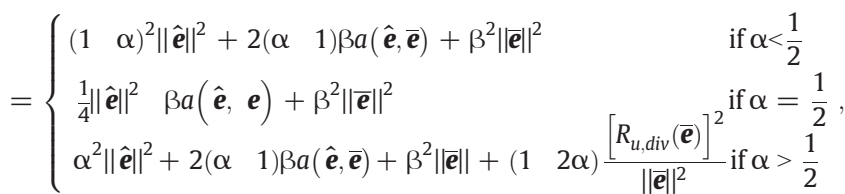

where we made use of the inequalities

$\frac{\left[R_{u, \mathrm{div}}(\overline{\boldsymbol{e}})\right]^{2}}{\|\overline{\boldsymbol{e}}\|^{2}} \leq\left\|\boldsymbol{e}_{0}\right\|^{2} \leq\|\hat{\boldsymbol{e}}\|^{2}$.

Evaluating the variation of these quadratic bounds gives us the optimal choice of $\alpha$ and $\beta$ that minimize $\Delta E$. There is no stationary point for either $\alpha<1 / 2$ or $\alpha>1 / 2$. The minimizer of $\Delta E$ has the expression

$\alpha=\frac{1}{2}$

$\beta=\frac{a(\hat{\boldsymbol{e}}, \overline{\boldsymbol{e}})}{2\|\overline{\boldsymbol{e}}\|^{2}}$

where $\beta$ was optimized given $\alpha=\frac{1}{2}$. Hence we may conclude the optimal bounds as follows:

$\bar{E}-\frac{\Delta E}{2} \leq\|\boldsymbol{e}\| \leq \bar{E}+\frac{\Delta E}{2}$

$\bar{E}=\left\|\boldsymbol{e}_{\mathrm{div}}+\frac{1}{2} \hat{\boldsymbol{e}}+\frac{1}{2} \frac{R_{u, \mathrm{div}}(\overline{\boldsymbol{e}})}{\|\overline{\boldsymbol{e}}\|^{2}} \overline{\boldsymbol{e}}\right\|$

$\Delta E=\sqrt{\|\hat{\boldsymbol{e}}\|^{2}-\frac{\left[R_{u, d i v}(\overline{\boldsymbol{e}})\right]^{2}}{\|\overline{\boldsymbol{e}}\|^{2}},}$

where we made use of the equality $a(\hat{\boldsymbol{e}}, \overline{\boldsymbol{e}})=R_{u, \operatorname{div}}(\overline{\boldsymbol{e}})$.

\section{Goal-oriented error estimation using the flux-free error estimator}

We now consider the case that we want to estimate the error in a linear output functional of the solution. Assume that the goal of our computation is to estimate the output functional defined by

$s=l_{u}^{*}(\boldsymbol{u})+l_{p}^{*}(p)$,

where $l_{u}^{*}$ and $l_{p}^{*}$ are linear functionals on $\mathcal{U}$ and $\mathcal{Q}$, respectively. From our FE solution, we thus obtain the approximation

$s_{H}=l_{u}^{*}\left(\boldsymbol{u}_{H}\right)+l_{p}^{*}\left(p_{H}\right)$

\subsection{The dual problem}

In order to proceed, we now define the dual problem pertinent to the chosen output as that of finding $\left(\boldsymbol{u}^{*}, p^{*}\right) \in \mathcal{V} \times \mathcal{Q}$ such that

$a\left(\boldsymbol{w}, \boldsymbol{u}^{*}\right)+b\left(p^{*}, \boldsymbol{w}\right)=l_{u}^{*}(\boldsymbol{w}) \quad \forall \boldsymbol{w} \in \mathcal{V}$

$b\left(q, \boldsymbol{u}^{*}\right)=l_{p}^{*}(q) \quad \forall q \in \mathcal{Q}$, 
where we note the similarity to the original problem Eqs. (6) and (7), henceforth denoted the primal problem.

Remark. Here, we assume that $l_{u}^{*}$ acts as a linear functional on $\mathcal{V}$ as well as $\mathcal{U}$, i.e., we assume that

$l_{u}^{*}(\boldsymbol{v}+\boldsymbol{w})=l_{u}^{*}(\boldsymbol{v})+l_{u}^{*}(\boldsymbol{w}) \quad \forall \boldsymbol{v}, \boldsymbol{w} \in \mathcal{U} \times \mathcal{V}$.

Similarly as for the primal problem, we start off by computing the approximations $\left(\boldsymbol{u}_{H}^{*}, p_{H}^{*}\right) \in \mathcal{V}_{H} \times \mathcal{Q}_{H}$ such that

$a\left(\boldsymbol{w}, \boldsymbol{u}_{H}^{*}\right)+b\left(p_{H}^{*}, \boldsymbol{w}\right)=l_{u}^{*}(\boldsymbol{w}) \quad \forall \boldsymbol{w} \in \mathcal{V}_{H}$

$b\left(q, \boldsymbol{u}_{H}^{*}\right)=l_{p}^{*}(q) \quad \forall q \in \mathcal{Q}_{H}$,

whereby we are in the position to define the dual residuals as

$R_{u}^{*}(\boldsymbol{w}):=l_{u}^{*}(\boldsymbol{w})-a\left(\boldsymbol{w}, \boldsymbol{u}_{H}^{*}\right)-b\left(p_{H}^{*}, \boldsymbol{w}\right)$,

$R_{p}^{*}(q):=l_{p}^{*}(q)-b\left(q, \boldsymbol{u}_{H}^{*}\right)$

We now utilize the split of the error into the divergence part $\left(\boldsymbol{e}_{\mathrm{div}}^{*}\right.$, $\left.g_{\text {div }}^{*}\right)$ and a divergence free part $\left(\boldsymbol{e}_{0}^{*}, g_{0}^{*}\right)$ as follows:

$\boldsymbol{e}^{*}:=\boldsymbol{u}^{*}-\boldsymbol{u}_{H}^{*}=\boldsymbol{e}_{\mathrm{div}}^{*}+\boldsymbol{e}_{0}^{*}$

$g^{*}:=p^{*}-p_{H}^{*}=g_{\text {div }}^{*}+g_{0}^{*}$

Following the steps described in Section 5, we now proceed by computing the equilibrated and divergence prescribed solution $\left(\boldsymbol{e}_{\mathrm{div}}^{*}\right.$, $g_{\text {div }}^{*}$ ) that satisfies the conditions

$a\left(\boldsymbol{w}, \boldsymbol{e}_{\text {div }}^{*}\right)-b\left(g_{\text {div }}^{*}, \boldsymbol{w}\right)=0 \quad \forall \boldsymbol{w} \in \mathcal{V}_{H, 1}$

$b\left(q, \boldsymbol{e}_{\mathrm{div}}^{*}\right)=R_{p}^{*}(q) \quad \forall q \in \mathcal{Q}$,

whereby we introduce the updated, divergence free, dual residuals

$R_{u, \operatorname{div}}^{*}(\boldsymbol{w}):=R_{u, \operatorname{div}}^{*}(\boldsymbol{w})-a\left(\boldsymbol{w}, \boldsymbol{e}_{\mathrm{div}}^{*}\right)-b\left(g_{\mathrm{div}}^{*}, \boldsymbol{w}\right)$,

$R_{p}^{*}(q):=l_{p}^{*}(q)-b\left(q, \boldsymbol{u}_{H}^{*}\right)$

The pertinent error equation for the divergence free part of the dual error may now be stated as

$a\left(\boldsymbol{w}, \boldsymbol{e}_{0}^{*}\right)+b\left(g_{0}^{*}, \boldsymbol{w}\right)=R_{u, \operatorname{div}}^{*}(\boldsymbol{w}) \quad \forall \boldsymbol{w} \in \mathcal{V}$,

$b\left(q, \boldsymbol{e}_{0}^{*}\right)=0 \quad \forall q \in \mathcal{Q}$

where $\left(\boldsymbol{e}_{0}^{*}, g_{0}^{*}\right) \in \mathcal{V} \times \mathcal{Q}$.

\subsubsection{Pressure output for constrained domain}

In the case of pure Dirichlet boundary conditions, as discussed in Section 2, we recall that the piecewise constant is removed from the pressure test and trial space as

$\mathcal{Q}^{\prime}=\left\{q \in L_{2}(\Omega) ; \quad \int_{\Omega} q d \omega=0\right\}$.

Hence, we must require that Eq. (149) holds for a piecewise constant test function, i.e., we require that

$l_{p}^{*}(1)=0$.
Considering the chosen pressure output $\hat{l}_{p}^{*}(q)$, we can always construct the pertinent equilibrated output functional as follows:

$l_{p}^{*}(q)=\hat{l}_{p}^{*}(q)-\frac{\hat{l}_{p}^{*}(1)}{\Omega} \int_{\Omega} q d \Omega$

The modified output is an identical functional on $\mathcal{Q}$ since

$l_{p}^{*}(q)=\hat{l}_{p}^{*}(q)-\frac{\hat{l}_{p}^{*}(1)}{|\Omega|} \underbrace{\int_{\Omega} q d \Omega}_{0}=\hat{l}_{p}^{*}(q) \quad \forall q \in \mathcal{Q}$,

and equilibrated since

$l_{p}^{*}(1)=\hat{l}_{p}^{*}(1)-\frac{\hat{l}_{p}^{*}(1)}{|\Omega|} \underbrace{\int_{\Omega} 1 d \Omega \equiv 0 .}_{|\Omega|}$

\subsection{Output representation}

We are now in the position of stating the representation of the output as follows:

$s=l_{u}^{*}(\boldsymbol{u})+l_{p}^{*}(p)=\underbrace{l_{u}^{*}\left(\boldsymbol{u}_{H}\right)+l_{p}^{*}\left(p_{H}\right)}_{: s_{H}}+l_{u}^{*}(\mathbf{e})+l_{p}^{*}(g)$

where we used the linearity of $l_{u}^{*}$ and $l_{p}^{*}$ and introduced the split of the primal solution into its FE part and its error. Furthermore, we introduced the output of the FE solution, $s_{H}$. Next, we use the formulation of the dual problem in Eqs. (145) and (146), together with the primal Galerkin orthogonality in Eqs. (36) and (37), to obtain

$$
\begin{aligned}
s & =s_{H}+a\left(\boldsymbol{e}, \boldsymbol{u}^{*}\right)+b\left(p^{*}, \boldsymbol{e}\right)+b\left(g, \boldsymbol{u}^{*}\right) \\
& =s_{H}+\underbrace{a\left(\boldsymbol{e}, \boldsymbol{u}_{H}^{*}\right)+b\left(p_{H}^{*}, \boldsymbol{e}\right)+b\left(g, \boldsymbol{u}_{H}^{*}\right)}_{0}+a\left(\boldsymbol{e}, \boldsymbol{e}^{*}\right)+b\left(g^{*}, \boldsymbol{e}\right)+b\left(g, \boldsymbol{e}^{*}\right) \\
& =s_{H}+a\left(\boldsymbol{e}, \boldsymbol{e}^{*}\right)+b\left(g^{*}, \boldsymbol{e}\right)+b\left(g, \boldsymbol{e}^{*}\right),
\end{aligned}
$$

where we made use of the split of the dual solution into its FE approximation and its error. Finally, we introduce the split of the errors into divergence and divergence free parts to obtain

$$
\begin{aligned}
s= & s_{H}+a\left(\boldsymbol{e}_{0}, \boldsymbol{e}_{0}^{*}\right)+a\left(\boldsymbol{e}_{\mathrm{div}}, \boldsymbol{e}_{0}^{*}\right)+a\left(\boldsymbol{e}_{0}, \boldsymbol{e}_{\mathrm{div}}^{*}\right)+a\left(e_{\mathrm{div}}, \boldsymbol{e}_{\mathrm{div}}^{*}\right) \\
& +\underbrace{b\left(g^{*}, \boldsymbol{e}_{0}\right)}_{0}+b\left(g_{0}^{*}, \boldsymbol{e}_{\mathrm{div}}\right)+b\left(g_{\mathrm{div}}^{*}, \boldsymbol{e}_{\mathrm{div}}\right) \\
& +\underbrace{b\left(g^{*}, \boldsymbol{e}_{0}\right)}_{0}+b\left(g_{0}, \boldsymbol{e}_{\mathrm{div}}^{*}\right)+b\left(g_{\mathrm{div}}, \boldsymbol{e}_{\mathrm{div}}^{*}\right) \\
= & s_{H}+a\left(\boldsymbol{e}_{0}, \boldsymbol{e}_{0}^{*}\right)+R_{u, \mathrm{div}}^{*}\left(\boldsymbol{e}_{\mathrm{div}}\right)+R_{u, \mathrm{div}}\left(\boldsymbol{e}_{\mathrm{div}}^{*}\right) \\
& +a\left(\boldsymbol{e}_{\mathrm{div}}, \boldsymbol{e}_{\mathrm{div}}^{*}\right)+b\left(g_{\mathrm{div}}^{*}, \boldsymbol{e}_{\mathrm{div}}\right)+b\left(g_{\mathrm{div}}, \boldsymbol{e}_{\mathrm{div}}^{*}\right),
\end{aligned}
$$

where we used the fact that $\boldsymbol{e}_{0}, \boldsymbol{e}_{0}^{*}$ are divergence free and identified the primal and dual error equations for the divergence free errors in Eqs. (45) and (46) and Eqs. (175) and (176), respectively.

In summary, we have the exact representation of the output as

$$
s=s_{H}+s_{\mathrm{div}}+s_{0}
$$

where $s_{H}$ and $s_{\text {div }}$ are computable,

$$
\begin{aligned}
s_{H}:= & l_{u}^{*}\left(\boldsymbol{u}_{H}\right)+l_{p}^{*}\left(p_{H}\right), \\
s_{\mathrm{div}}:= & R_{u, \mathrm{div}}^{*}\left(\boldsymbol{e}_{\mathrm{div}}\right)+R_{u, \mathrm{div}}\left(\boldsymbol{e}_{\mathrm{div}}^{*}\right) \\
& +a\left(\boldsymbol{e}_{\mathrm{div}}, \boldsymbol{e}_{\mathrm{div}}^{*}\right)+b\left(g_{\mathrm{div}}^{*}, \boldsymbol{e}_{\mathrm{div}}\right)+b\left(g_{\mathrm{div}}, \boldsymbol{e}_{\mathrm{div}}^{*}\right),
\end{aligned}
$$


and $s_{0}$ is the remaining unknown part,

$s_{0}:=a\left(\boldsymbol{e}_{0}, \boldsymbol{e}_{0}^{*}\right)$.

We shall now use the results obtained earlier for bounding $\left\|\boldsymbol{e}_{0}\right\|$ to bound the unknown term $s_{0}$.

Remark. An alternative format of Eq. (170) is

$s_{\mathrm{div}}=R_{u}^{*}\left(\boldsymbol{e}_{\mathrm{div}}\right)+R_{u}\left(\boldsymbol{e}_{\mathrm{div}}^{*}\right)-a\left(\boldsymbol{e}_{\mathrm{div}}, \boldsymbol{e}_{\mathrm{div}}^{*}\right)$,

whereby it is apparent that $g_{\text {div }}$ and $g_{\text {div }}^{*}$ do not affect $s_{\text {div }}$.

\subsection{Computation of bounds}

We shall now compute bounds for the unknown part of the output in the representation. Using the parallelogram law, we obtain the following equality:

$s_{0}=a\left(\boldsymbol{e}_{0}, \boldsymbol{e}_{0}^{*}\right)=a\left(\kappa \boldsymbol{e}_{0}, \frac{1}{\kappa} \boldsymbol{e}_{0}^{*}\right)=\frac{1}{4}\left\|z_{0}^{+}\right\|^{2}-\frac{1}{4}\left\|z_{0}\right\|^{2}$,

for any $\kappa \neq 0$. Here we introduced

$\boldsymbol{z}_{0}^{ \pm}:=\kappa \boldsymbol{e}_{0} \pm \frac{1}{\kappa} \boldsymbol{e}_{0}^{*}$,

as the combined primal/dual solution. In fact, since the linearity of the problem gives the pertinent error equation

$a\left(\boldsymbol{w}, \boldsymbol{z}_{0}^{ \pm}\right)+b\left(y_{0}^{ \pm}, \boldsymbol{w}\right)=R^{ \pm}(\boldsymbol{w}) \quad \forall \boldsymbol{w} \in \mathcal{V}$,

$b\left(q, \boldsymbol{z}_{0}^{ \pm}\right)=0 \quad \forall q \in \mathcal{Q}$,

where we introduced the combined primal/dual residual and error in pressure as

$R^{ \pm}(\boldsymbol{w}):=\kappa R_{u, \mathrm{div}}(\boldsymbol{w}) \pm \frac{1}{\kappa} R_{u, \mathrm{div}}^{*}(\boldsymbol{w}), \quad y_{0}^{ \pm}:=\kappa g_{0} \pm \frac{1}{\kappa} g_{0}^{*}$,

we may use the strategy presented in Section 6.2 to bound $\left\|\boldsymbol{z}_{0}^{ \pm}\right\|$from above and below. Hence, again following from linearity of the problem, we may add up

$\hat{\boldsymbol{z}}^{ \pm}=\kappa \hat{\boldsymbol{e}} \pm \frac{1}{\kappa} \hat{\boldsymbol{e}}^{*}$,

such that

$\left\|\boldsymbol{z}_{0}^{ \pm}\right\|^{2} \leq\left\|\boldsymbol{z}_{0}^{ \pm}\right\|_{\mathrm{UB}}^{2}$

where

$\left\|z_{0}^{ \pm}\right\|_{\mathrm{UB}}^{2}:=\left\|z_{0}^{ \pm}\right\|^{2}$.

Turning to the lower bound, assuming that we have computed the solutions $\overline{\boldsymbol{e}}_{\mathrm{dev}}$ and $\overline{\boldsymbol{e}}_{\mathrm{dev}}^{*}$ pertinent to un equilibrated local Dirichlet problems, cf. Section 5 , we note that we now have a two dimensional (linear) set for solutions $\overline{\boldsymbol{z}}^{ \pm} \in \mathcal{V}$ that fulfill the incompressibility condition, namely

$$
\overline{\boldsymbol{z}}^{ \pm}=\alpha^{ \pm} \overline{\boldsymbol{e}}+\alpha^{* \pm} \overline{\boldsymbol{e}}^{*}
$$

Hence, we formulate the lower bound as

$\left\|\boldsymbol{z}_{0}^{ \pm}\right\|^{2} \geq\left\|\boldsymbol{z}_{0}^{ \pm}\right\|_{\mathrm{LB}}^{2}$ where

$$
\begin{aligned}
\left\|\boldsymbol{z}_{0}^{ \pm}\right\|_{\mathrm{LB}}^{2}= & \max _{\alpha^{ \pm}, \alpha^{* \pm}}\left\{2 R_{u, \operatorname{div}}^{ \pm}\left(\alpha^{ \pm} \overline{\boldsymbol{e}}+\alpha^{* \pm} \overline{\boldsymbol{e}}^{*}\right)-\left\|\alpha^{ \pm} \overline{\boldsymbol{e}}+\alpha^{* \pm} \overline{\boldsymbol{e}}^{*}\right\|^{2}\right\} \\
= & \max _{\alpha^{ \pm}, \alpha^{* \pm}}\left\{2 \alpha^{ \pm} R_{u, \operatorname{div}}^{ \pm}(\overline{\boldsymbol{e}})+2 \alpha^{* \pm} R_{u, \operatorname{div}}^{ \pm}\left(\overline{\boldsymbol{e}}^{*}\right)\right. \\
& \left.-\left(\alpha^{ \pm}\right)^{2}\|\overline{\boldsymbol{e}}\|^{2}-2 \alpha^{ \pm} \alpha^{* \pm} a\left(\overline{\boldsymbol{e}}, \overline{\boldsymbol{e}}^{*}\right)-\left(\alpha^{*^{ \pm}}\right)^{2}\left\|\overline{\boldsymbol{e}}^{*}\right\|^{2}\right\} .
\end{aligned}
$$

We expect $\alpha^{ \pm}=\kappa$ and $\alpha^{*} \pm= \pm 1 / \kappa$ to be good approximations (corresponding to the candidates $\left.\kappa \overline{\boldsymbol{e}} \pm(1 / \kappa) \overline{\boldsymbol{e}}^{*}\right)$. However, since we have full liberty to choose the parameters, we simply maximize the lower bound. For any case when $\left|a\left(\overline{\boldsymbol{e}}, \overline{\boldsymbol{e}}^{*}\right)\right|<\|\overline{\boldsymbol{e}}\|\left\|\overline{\boldsymbol{e}}^{*}\right\|$, thus corresponding to $\overline{\boldsymbol{e}}$ and $\overline{\boldsymbol{e}}^{*}$ not being linearly dependent, we obtain the maximizing parameters by solving the linear problem pertinent to the stationary point for the (quadratic) minimization problem, i.e.,

$\left[\begin{array}{lc}\|\bar{e}\|^{2} & a\left(\overline{\boldsymbol{e}}, \overline{\boldsymbol{e}}^{*}\right) \\ a\left(\overline{\boldsymbol{e}}, \overline{\boldsymbol{e}}^{*}\right) & \left\|\overline{\boldsymbol{e}}^{*}\right\|^{2}\end{array}\right]\left[\begin{array}{c}\alpha^{ \pm} \\ \alpha^{* \pm}\end{array}\right]=\left[\begin{array}{c}R_{u, \mathrm{div}}^{ \pm}(\overline{\boldsymbol{e}}) \\ R_{u, \mathrm{div}}^{ \pm}\left(\overline{\boldsymbol{e}}^{*}\right)\end{array}\right]$.

The analytical solution to Eq. (184) can be found independently of $\kappa$ such that

$\left\|z_{0}^{ \pm}\right\|_{L \mathrm{~B}}^{2}=\frac{\|\overline{\boldsymbol{e}}\|^{2}\left(R_{u, \mathrm{div}}^{ \pm}\left(\overline{\boldsymbol{e}}^{*}\right)\right)^{2}-2 a\left(\overline{\boldsymbol{e}}, \overline{\boldsymbol{e}}^{*}\right) R_{u, \mathrm{div}}^{ \pm}(\overline{\boldsymbol{e}}) R_{u, d i v}^{ \pm}\left(\overline{\boldsymbol{e}}^{*}\right)+\left\|\left.\right|^{*}\right\|^{2}\left(R_{u, d i v}^{ \pm}(\overline{\boldsymbol{e}})\right)^{2}}{\|\overline{\boldsymbol{e}}\|^{2}\left\|\left.\right|^{*}\right\|^{2}-\left(a\left(\overline{\boldsymbol{e}}, \overline{\boldsymbol{e}}^{*}\right)\right)^{2}}$.

Rearranging the terms, we can show that $\left\|\boldsymbol{z}_{0}^{ \pm}\right\|_{\mathrm{LB}}$ is sharper estimate than the trivial one using that the evaluated residuals are scalars such that

$$
\begin{aligned}
& \left\|\boldsymbol{z}_{0}^{ \pm}\right\|_{\mathrm{LB}}^{2}=\frac{\left.a\left(e \cdot R_{u, \mathrm{div}}^{ \pm}\left(\overline{\boldsymbol{e}}^{*}\right)-\overline{\boldsymbol{e}}^{*} \cdot R_{u, \operatorname{div}}^{ \pm}(\overline{\boldsymbol{e}}), \overline{\boldsymbol{e}} \cdot R_{u, \operatorname{div}}^{ \pm}\left(\overline{\boldsymbol{e}}^{*}\right)-\overline{\boldsymbol{e}}^{*} \cdot R_{u, \operatorname{div}}^{ \pm}(\overline{\boldsymbol{e}})\right)\right)}{\|\overline{\boldsymbol{e}}\|^{2}\left\|\overline{\boldsymbol{e}}^{*}\right\|^{2}-\left(a\left(\overline{\boldsymbol{e}}, \overline{\boldsymbol{e}}^{*}\right)\right)^{2}} \\
& =\frac{\left.\| \overline{\boldsymbol{e}} \cdot R_{u, \operatorname{div}}^{ \pm}\left(\overline{\boldsymbol{e}}^{*}\right)-\overline{\boldsymbol{e}}^{*} \cdot R_{u, d i v}^{ \pm}(\overline{\boldsymbol{e}})\right) \|^{2}}{\|\overline{\boldsymbol{e}}\|^{2}\left\|\overline{\boldsymbol{e}}^{*}\right\|^{2}\left(a\left(\overline{\boldsymbol{e}} \overline{\boldsymbol{e}}^{*}\right)\right)^{2}} \geq 0 .
\end{aligned}
$$

Returning to the special case of $\overline{\boldsymbol{e}}$ and $\overline{\boldsymbol{e}}^{*}$ being linearly dependent, i.e. that $\overline{\boldsymbol{e}}=\alpha \overline{\boldsymbol{e}}^{*}$ for some scalar $\alpha$, we note that the maximization problem is one dimensional, whereby we obtain the result

$\left\|\boldsymbol{z}_{0}^{ \pm}\right\|_{\mathrm{LB}}^{2}=\frac{\left(R_{u, \mathrm{div}}^{ \pm}(\overline{\boldsymbol{e}})\right)^{2}}{\|\overline{\boldsymbol{e}}\|^{2}}=\frac{\left(R_{u, \mathrm{div}}^{ \pm}\left(\overline{\boldsymbol{e}}^{*}\right)\right)^{2}}{\left\|\overline{\boldsymbol{e}}^{*}\right\|^{2}}$.

Remark. As for the case of the energy norm evaluation, the lower bound can be further optimized in the case that the pressure approximation is discontinuous. In that case the local functions $\overline{\boldsymbol{e}}_{k}$ and $\overline{\boldsymbol{e}}_{k}^{*}$ can be varied independently, whereby we obtain the results

$\left\|z_{0}^{ \pm}\right\|_{\mathrm{LB}}^{2}=\sum_{k}^{N E L} \eta_{k}^{2}$

where

$\eta_{k}^{2}= \begin{cases}\frac{\left(R_{u, d i v, k}^{ \pm}\left(\overline{\boldsymbol{e}}_{k}\right)\right)^{2}}{\left\|\overline{\boldsymbol{e}}_{k}\right\|_{\Omega_{k}}^{2}} & \text { if } \overline{\boldsymbol{e}}_{k}=\beta \overline{\boldsymbol{e}}_{k}^{*} \text { for any } \beta \in \mathbb{R} \\ \frac{\left\|\overline{\boldsymbol{e}}_{k} \cdot R_{u, \mathrm{div}, k}^{ \pm}\left(\overline{\boldsymbol{e}}_{k}^{*}\right)-\overline{\boldsymbol{e}}_{k}^{*} \cdot R_{u, \mathrm{div}, k}^{ \pm}\left(\overline{\boldsymbol{e}}_{k}\right)\right\|_{\Omega_{k}}^{2}}{\left\|\overline{\boldsymbol{e}}_{k}\right\|_{\Omega_{k}}^{2}\left\|\overline{\boldsymbol{e}}_{k}^{*}\right\|_{\Omega_{k}}^{2}-\left(a_{k}\left(\overline{\boldsymbol{e}}_{k}, \overline{\boldsymbol{e}}_{k}^{*}\right)\right)^{2}} & \text { else. }\end{cases}$ 
In order to bound $s_{0}$, we may now use Eqs. (173) and (179) to obtain

$\frac{1}{4}\left\|z_{0}^{+}\right\|_{\mathrm{LB}}^{2}-\frac{1}{4}\left\|\boldsymbol{z}_{0}\right\|_{\mathrm{UB}}^{2} \leq s_{0} \leq \frac{1}{4}\left\|\boldsymbol{z}_{0}^{+}\right\|_{\mathrm{UB}}^{2}-\frac{1}{4}\left\|\boldsymbol{z}_{0}\right\|_{\mathrm{LB}}^{2}$

or, in terms of error bounds,

$\bar{s}-\frac{1}{2} \Delta s \leq s \leq \bar{s}+\frac{1}{2} \Delta s$,

where $\bar{s}$ is the average (expected) value of the output and $\Delta s$ is the bound gap,

$\bar{s}:=s_{H}+s_{\mathrm{div}}+\frac{1}{8}\left(\left\|z_{0}^{+}\right\|_{\mathrm{UB}}^{2}+\left\|\boldsymbol{z}_{0}^{+}\right\|_{\mathrm{LB}}^{2}-\left\|\boldsymbol{z}_{0}\right\|_{\mathrm{UB}}^{2}-\left\|\boldsymbol{z}_{0}\right\|_{\mathrm{LB}}^{2}\right)$,

$\Delta s:=\frac{1}{4}\left(\left\|z_{0}^{+}\right\|_{\mathrm{UB}}^{2}-\left\|z_{0}^{+}\right\|_{\mathrm{LB}}^{2}+\left\|\boldsymbol{z}_{0}\right\|_{\mathrm{UB}}^{2}-\left\|\boldsymbol{z}_{0}\right\|_{\mathrm{LB}}^{2}\right)$.

\subsection{Optimization of the bound gap}

Finally, we recall that the parameter $\kappa$, defining $\boldsymbol{z}^{ \pm}$, can be chosen arbitrarily. Hence, we wish to choose $\kappa$ such that the bound gap $\Delta s$ is minimized.

First, we shall expand the upper bound estimates in terms of $\kappa$,

$\left\|\boldsymbol{z}_{0}^{ \pm}\right\|_{\text {UB }}^{2}=a\left(\kappa \hat{\boldsymbol{e}} \pm \frac{1}{\kappa} \hat{\boldsymbol{e}}^{*}, \kappa \hat{\boldsymbol{e}} \pm \frac{1}{\kappa} \hat{\boldsymbol{e}}^{*}\right)=\kappa^{2}\|\hat{\boldsymbol{e}}\|^{2} \pm 2 a\left(\hat{\boldsymbol{e}}, \hat{\boldsymbol{e}}^{*}\right)+\frac{1}{\kappa}\left\|\hat{\boldsymbol{e}}^{2}\right\|$.

Turning to the lower bound, we shall now derive the results for three special cases below:

\subsubsection{Optimized bounds for no improved solutions}

For the case that we have not computed $\overline{\boldsymbol{e}}$ and $\overline{\boldsymbol{e}}^{*}$, we use the trivial bounds

$\left\|z_{0}^{ \pm}\right\|_{L B}^{2}=0$

whereby we can express the bound center and gap as

$\bar{s}=s_{H}+s_{\mathrm{div}}+\frac{1}{2} a\left(\hat{\boldsymbol{e}}, \hat{\boldsymbol{e}}^{*}\right)$,

$\Delta s=\frac{1}{2}\left(\kappa^{2}\|\hat{\boldsymbol{e}}\|^{2}+\frac{1}{\kappa^{2}}\left\|\hat{\boldsymbol{e}}^{*}\right\|^{2}\right)$

The bound cap can be minimized choosing $k^{2}=\left\|\hat{\boldsymbol{e}}^{*}\right\| /\|\hat{\boldsymbol{e}}\|$, whereby we obtain the final result

$s:=\bar{s}-\frac{1}{2} \Delta s \leq s \leq \bar{s}+\frac{1}{2} \Delta s=: s^{+}$,

$\bar{s}=s_{H}+s_{\mathrm{div}}+\frac{1}{2} a\left(\hat{\boldsymbol{e}}, \hat{\boldsymbol{e}}^{*}\right)$,

$\Delta s=\|\hat{\boldsymbol{e}}\|\left\|\hat{\boldsymbol{e}}^{*}\right\|$.
8.4.2. Optimized bounds utilizing improved solutions

As shown previously, computation of improved solutions $\overline{\boldsymbol{e}}$ and $\overline{\boldsymbol{e}}^{*}$ improve the bound. Expanding the lower bounds Eq. (187) in terms of $\kappa$ gives

$$
\begin{aligned}
\left\|z_{0}^{ \pm}\right\|_{\mathrm{LB}}^{2}= & \kappa^{2} \frac{\delta\left(\overline{\boldsymbol{e}}, \overline{\boldsymbol{e}}^{*}\right)}{\gamma\left(\overline{\boldsymbol{e}}, \overline{\boldsymbol{e}}^{*}\right)}+\frac{1}{\kappa^{2}} \frac{\delta^{*}\left(\overline{\boldsymbol{e}}, \overline{\boldsymbol{e}}^{*}\right)}{\gamma\left(\overline{\boldsymbol{e}}, \overline{\boldsymbol{e}}^{*}\right)} \\
& \pm 2 \frac{\|\overline{\boldsymbol{e}}\|^{2} R_{u, \operatorname{div}}\left(\overline{\boldsymbol{e}}^{*}\right) R_{u, \operatorname{div}}^{*}\left(\overline{\boldsymbol{e}}^{*}\right)}{\gamma\left(\overline{\boldsymbol{e}}, \overline{\boldsymbol{e}}^{*}\right)} \\
& \mp 2 \frac{a\left(\overline{\boldsymbol{e}}, \overline{\boldsymbol{e}}^{*}\right)\left(R_{u, \operatorname{div}}(\overline{\boldsymbol{e}}) R_{u, \operatorname{div}}^{*}\left(\overline{\boldsymbol{e}}^{*}\right)+R_{u, \operatorname{div}}^{*}(\overline{\boldsymbol{e}}) R_{u, \operatorname{div}}\left(\overline{\boldsymbol{e}}^{*}\right)\right)}{\gamma\left(\overline{\boldsymbol{e}}, \overline{\boldsymbol{e}}^{*}\right)} \\
& \pm 2 \frac{\left\|\overline{\boldsymbol{e}}^{*}\right\|^{2} R_{u, \operatorname{div}}(\overline{\boldsymbol{e}}) R_{u, \operatorname{div}}^{*}(\overline{\boldsymbol{e}})}{\gamma\left(\overline{\boldsymbol{e}}, \overline{\boldsymbol{e}}^{*}\right)},
\end{aligned}
$$

where we introduce the abbreviating forms

$$
\begin{aligned}
\gamma\left(\overline{\boldsymbol{e}}, \overline{\boldsymbol{e}}^{*}\right):= & \|\overline{\boldsymbol{e}}\|^{2}\left\|\overline{\boldsymbol{e}}^{*}\right\|^{2}-\left(a\left(\overline{\boldsymbol{e}}, \overline{\boldsymbol{e}}^{*}\right)\right)^{2}, \\
\delta\left(\overline{\boldsymbol{e}}, \overline{\boldsymbol{e}}^{*}\right):= & \|\overline{\boldsymbol{e}}\|^{2}\left(R_{u, \operatorname{div}}\left(\overline{\boldsymbol{e}}^{*}\right)\right)^{2}+\left\|\overline{\boldsymbol{e}}^{*}\right\|^{2}\left(R_{u, \operatorname{div}}(\overline{\boldsymbol{e}})\right)^{2} \\
& -2 a\left(\overline{\boldsymbol{e}}, \overline{\boldsymbol{e}}^{*}\right) R_{u, \operatorname{div}}(\overline{\boldsymbol{e}}) R_{u, \operatorname{div}}\left(\overline{\boldsymbol{e}}^{*}\right), \\
\delta^{*}\left(\overline{\boldsymbol{e}}, \overline{\boldsymbol{e}}^{*}\right):= & \|\overline{\boldsymbol{e}}\|^{2}\left(R_{u, \operatorname{div}}^{*}\left(\overline{\boldsymbol{e}}^{*}\right)\right)^{2}+\left\|\overline{\boldsymbol{e}}^{*}\right\|^{2}\left(R_{u, \operatorname{div}}^{*}(\overline{\boldsymbol{e}})\right)^{2} \\
& -2 a\left(\overline{\boldsymbol{e}}, \overline{\boldsymbol{e}}^{*}\right) R_{u, \operatorname{div}}^{*}(\overline{\boldsymbol{e}}) R_{u, \operatorname{div}}^{*}\left(\overline{\boldsymbol{e}}^{*}\right) .
\end{aligned}
$$

We can thus express the bound center and gap as

$$
\begin{aligned}
\bar{s}= & s_{H}+s_{\mathrm{div}}+\frac{1}{2} a\left(\hat{\boldsymbol{e}}, \hat{\boldsymbol{e}}^{*}\right) \\
& +\frac{\|\overline{\boldsymbol{e}}\|^{2} R_{u, \operatorname{div}}\left(\overline{\boldsymbol{e}}^{*}\right) R_{u, \operatorname{div}}^{*}\left(\overline{\boldsymbol{e}}^{*}\right)}{2 \gamma\left(\overline{\boldsymbol{e}}, \overline{\boldsymbol{e}}^{*}\right)}+\frac{\left\|\overline{\boldsymbol{e}}^{*}\right\|^{2} R_{u, \mathrm{div}}(\overline{\boldsymbol{e}}) R_{u, \operatorname{div}}^{*}(\overline{\boldsymbol{e}})}{2 \gamma\left(\overline{\boldsymbol{e}}, \overline{\boldsymbol{e}}^{*}\right)} \\
& -\frac{a\left(\overline{\boldsymbol{e}}, \overline{\boldsymbol{e}}^{*}\right)\left(R_{u, \operatorname{div}}(\overline{\boldsymbol{e}}) R_{u, \operatorname{div}}^{*}\left(\overline{\boldsymbol{e}}^{*}\right)+R_{u, \operatorname{div}}^{*}(\overline{\boldsymbol{e}}) R_{u, \operatorname{div}}\left(\overline{\boldsymbol{e}}^{*}\right)\right)}{2 \gamma\left(\overline{\boldsymbol{e}}, \overline{\boldsymbol{e}}^{*}\right)},
\end{aligned}
$$$$
\Delta s=\frac{\kappa^{2}}{2}\left(\|\hat{\boldsymbol{e}}\|^{2}-\frac{\delta\left(\overline{\boldsymbol{e}}, \overline{\boldsymbol{e}}^{*}\right)}{\gamma\left(\overline{\boldsymbol{e}}, \overline{\boldsymbol{e}}^{*}\right)}\right)+\frac{1}{2 \kappa^{2}}\left(\left\|\hat{\boldsymbol{e}}^{*}\right\|^{2}-\frac{\delta^{*}\left(\overline{\boldsymbol{e}}, \overline{\boldsymbol{e}}^{*}\right)}{\gamma\left(\overline{\boldsymbol{e}}, \overline{\boldsymbol{e}}^{*}\right)}\right) .
$$

Since we know that $\Delta s \geq 0$ for all $\kappa$, the two terms pertinent to $\kappa^{2}$ and $\kappa^{-2}$ must individually be $\geq 0$. If one term is 0 , this means that $s \equiv \bar{s}$. For positive terms, the bound cap can be minimized choosing

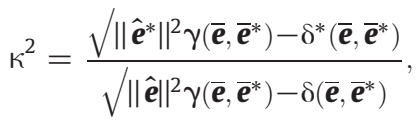

whereby we obtain the final result

$s:=\bar{s}-\frac{1}{2} \Delta s \leq s \leq s+\frac{1}{2} \Delta s=: s^{+}$,

$\bar{s}=s_{H}+s_{\mathrm{div}}+\frac{1}{2} a\left(\hat{\boldsymbol{e}}, \hat{\boldsymbol{e}}^{*}\right)$

$$
\begin{aligned}
& +\frac{\|\overline{\boldsymbol{e}}\|^{2} R_{u, \mathrm{div}}\left(\overline{\boldsymbol{e}}^{*}\right) R_{u, \mathrm{div}}^{*}\left(\overline{\boldsymbol{e}}^{*}\right)}{2 \gamma\left(\overline{\boldsymbol{e}}, \overline{\boldsymbol{e}}^{*}\right)}+\frac{\left\|\hat{\boldsymbol{e}}^{*}\right\|^{2} R_{u, \mathrm{div}}(\overline{\boldsymbol{e}}) R_{u, \mathrm{div}}^{*}(\overline{\boldsymbol{e}})}{2 \gamma\left(\overline{\boldsymbol{e}}, \overline{\boldsymbol{e}}^{*}\right)} \\
& -\frac{a\left(\overline{\boldsymbol{e}}, \overline{\boldsymbol{e}}^{*}\right)\left(R_{u, \operatorname{div}}(\overline{\boldsymbol{e}}) R_{u, \operatorname{div}}^{*}\left(\overline{\boldsymbol{e}}^{*}\right)+R_{u, \operatorname{div}}^{*}(\overline{\boldsymbol{e}}) R_{u, \operatorname{div}}\left(\overline{\boldsymbol{e}}^{*}\right)\right)}{2 \gamma\left(\overline{\boldsymbol{e}}, \overline{\boldsymbol{e}}^{*}\right)},
\end{aligned}
$$

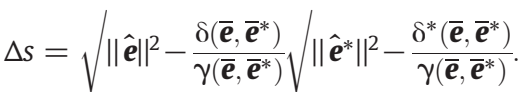




\subsection{Summary of the procedure for computing the bounds}

We now summarize the procedure for computing bounds for the quantity of interest,

$s=l_{u}^{*}(\boldsymbol{u})+l_{p}^{*}(p)$.

We split the computational procedure into subtasks as follows:

1. Find the approximate solution $\left(\boldsymbol{u}_{H}, p_{H}\right)$ from Eqs. (17) and (18) in Section 2 and compute the pertinent $\mathrm{FE}$ approximation $s_{H}$ from Eq. (144)

2. Find the dual solution $\left(\boldsymbol{u}_{H}^{*}, p_{H}^{*}\right)$ from Section 8, Eqs. (148) and (149).

3. Compute the divergence capturing error $\left(\boldsymbol{e}_{\text {div }}, g_{\text {div }}\right)$ and $\left(\boldsymbol{e}_{\text {div }}^{*}, g_{\text {div }}^{*}\right)$ in the dual and primal solutions, respectively, through the solution of local expanded Dirichlet problems as shown in Section 5, Eqs. (58) (60) (discontinuous pressure) or Eqs. (77) (79) and (83) (continuous pressure).

4. For the improved estimator, also solve for the unconstrained local Dirichlet problems resulting in $\left(\overline{\boldsymbol{e}}_{\text {div }}, \bar{g}_{\text {div }}\right)$ and $\left(\overline{\boldsymbol{e}}_{\text {div }}^{*}, \bar{g}_{\text {div }}^{*}\right)$ from Eqs. (51) and (52) (on elements for discontinuous pressure approximations) or Eqs. (72) and (73) (on stars for continuous pressure approximations).

5. Compute the broken approximation of the divergence free errors $(\hat{\boldsymbol{e}}, \hat{\mathrm{g}})$ and $\left(\hat{\boldsymbol{e}}^{*}, \hat{\mathrm{g}}^{*}\right)$ through the solution of local flux free problems on stars as described in Section 6, Eqs. (97) and (98).

6. Compute the optimal lower and upper bounds $s^{-}$and $s^{+}$, respectively, following the results in Section 8.4, from Eqs. (198) (200) (without the solution of unconstrained Dirichlet problems) or Eqs. (208) (210) (utilizing the solutions from the unconstrained local Dirichlet problems).

\section{Numerical examples}

In this section, we shall present two numerical examples, illustrating the behavior of the presented estimators. The chosen examples are both in 2D and we have limited the study to using triangular elements. The first example, Stokes' flow on the unit square, is a simple problem where the exact solution is known while the second example of Stokes' channel flow past a step obstacle shows the application towards less trivial flow patterns.

\subsection{Stokes' flow on the unit square}

As a first example, we shall study the Stokes problem on a unit square in $2 D,(x, y) \in[0,1]^{2}$ with Dirichlet boundary conditions. In order to investigate the accuracy and efficiency of the estimators, we consider a problem with known analytical solution. We show results for the following elements: The Mini element, the Taylor Hood element, the $\mathrm{P} 2 \mathrm{PO}$ element and the Crouxier Raviart element. In order to study the convergence of the bounds, we perform uniform refinement of an initial mesh. The local problems (of Dirichlet and flux free type) are solved in a manner such that each element $\Omega_{e}$ is subdivided into 100 new elements of the same type.

For known solution $(\boldsymbol{u}, p)$ on the unit square, we may compute the source term by use of Eqs. (1) (5) and prescribe the pertinent Dirichlet conditions. Two restrictions apply two the choice of $\boldsymbol{u}$ and $p$ : First, the velocity field must be divergence free, i.e., it must satisfy Eq. (2). Secondly, since we use solely Dirichlet boundary condition, the pressure must lie in $\mathcal{Q}^{\prime}$ defined in Eq. (14). It can easily be shown that velocity $\left(\boldsymbol{u}=\left(u_{x}, u_{y}\right)\right)$ and pressure fields on the form

$u_{x}=U(x) U^{\prime}(y)$,

$u_{y}=-U^{\prime}(x) U(y)$

$p=P(x, y)-\int_{\Omega} P(x, y) d \Omega$,

satisfy theses conditions for arbitrary choices of $U(\xi)$ and $P(x, y)$. For our numerical example, we choose

$U(\xi)=\xi^{4}-2 \xi^{3}+\xi^{2}$,

$P(x, y)=(x-.5)^{3}$,

whereby we ensure (i) that the solution is represented exactly on the boundary by any elements (homogeneous Dirichlet conditions) and (ii) that the exact solution is a polynomial of order 7 in velocity and a polynomial of order 3 in pressure, and can thus not be represented exactly by any of our chosen elements. In Fig. 1, the initial and the most refined mesh used in the convergence analysis are shown. An example of a numerical solution computed using Mini elements and 3683 degrees of freedom is illustrated in Fig. 2.

\subsubsection{Estimation of the error in velocity energy norm}

First, we consider the velocity energy norm of the total error, following the procedure described in Section 7. In Table 1, we show the numerical values for the computed upper and lower bounds of the velocity energy norm of the error using both the low cost bounds, cf. Eq. (128), and the more Accurate bounds using the improved Dirichlet problems, cf. Eq. (140). Since we study a problem where the analytical solution is known, we also show the exact value of the norm of the error for comparison. Note that, for the elements with discontinuous pressure approximation, the solutions with Eqs. (58) (60) and without Eqs. (51) and (52) the orthogonality constraint coincide,
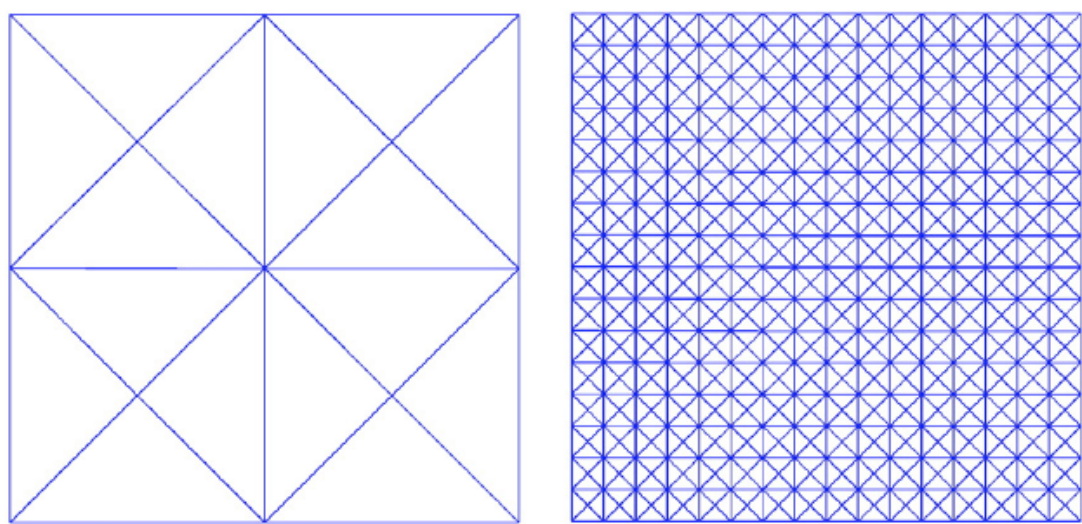

Fig. 1. Coarsest and finest mesh-triangulation used in the example of Stokes' flow on the unit square. The initial, coarsest, mesh (left) consists of 16 triangular elements, while the most refined mesh (right) consists of 1024 elements. 


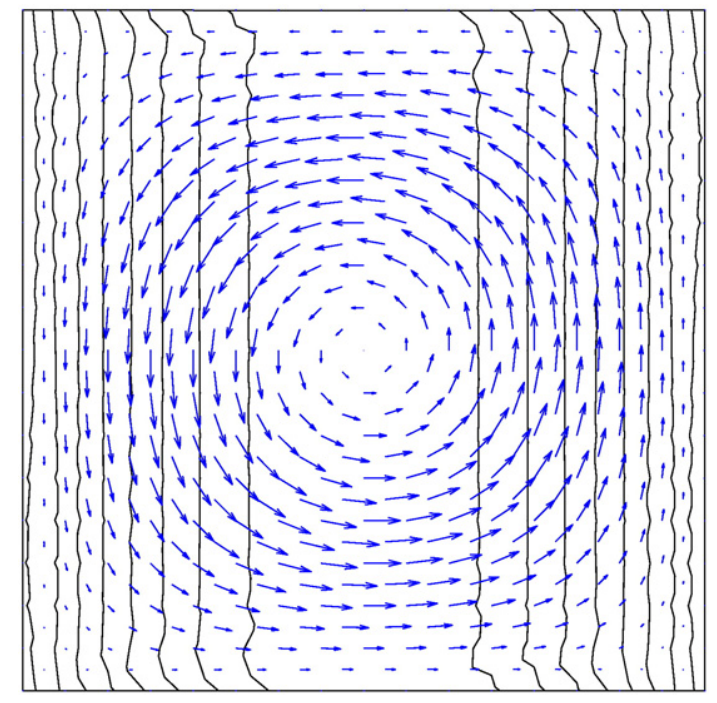

Fig. 2. A numerical solution to the example of Stokes' flow on the unit square. Velocities are shown together with iso-pressure lines. The approximation was obtained by using Mini-elements and 3683 degrees of freedom.

and thus no improved (Accurate) bound can be obtained. In Fig. 3, we show the exact value as well as the computed upper and lower bounds for the velocity energy norm of the error obtained by using the Mini elements. We note that the expected rates of convergence are obtained, i.e.,

$\|e\| \sim N^{r / 2} \sim h^{r}$

with $r=1$ for the Mini and P2P0 elements (1st order) and $r=2$ for the Taylor Hood and the Crouxier Raviart elements (2nd order).

\subsubsection{Estimation of the goal quantity}

We shall now investigate the behavior of the goal oriented error estimators presented in Section 8. To this end, we first choose the specified output to be that of the average velocity on the right half of the unit square, i.e.,

$s=\frac{1}{0.5} \int_{x>.5} \boldsymbol{u} \cdot \boldsymbol{e}_{y} d \Omega$,

where $\boldsymbol{e}_{y}$ is the unit vector aligned with the positive $y$ axis and the area of the considered region is $0.5 \times 1=0.5$. The exact value of the quantity can easily be computed from Eqs. (211) and (212) to be $s=1 / 240 \approx 0.00416667$. An example of the numerical approximation of the pertinent dual problem is shown in Fig. 4. In Table 2, we show the numerical values for the computed upper and lower bounds of the output using both the Low cost bounds, cf. Eq. (198), and the more Accurate bounds using the improved Dirichlet problems, cf. Eq. (208). As for the estimation of velocity energy error, no improved (Accurate) bounds can be obtained for the discontinuous pressure approximating elements. In Fig. 5, we show the computed upper and lower bounds (scaled by the exact value) for chosen goal quantity versus number of degrees of freedom for the four different element type discretizations discussed above. In Fig. 6 , the convergence of the bound gap, $\Delta s$, with mesh refinement is shown for the same case.

\subsubsection{Estimation of local goal quantities}

The goal quantity studied in the previous paragraph was defined on half the domain. Next, we consider the effect of considering a locally defined goal quantity. To this end we study an output on the form

$s=\frac{1}{\pi r^{2}} \int_{\left|x \quad x_{0}\right|<r} u \cdot e_{y} d \Omega$,

measuring the mean velocity in the $y$ direction inside a circular domain of radius $r$ centered at $\boldsymbol{x}_{0}=(0.75,0.50)$ where $\boldsymbol{x}=(x, y)$

Table 1

Exact values and computed (relative) bounds for the velocity-energy norm of the error in example of Stokes' flow on a unit square. Results are shown for both the Low cost method and the Accurate method, relying on an additional Dirichlet solution. Using $N$ refers to the total number of degrees of freedom.

\begin{tabular}{|c|c|c|c|c|c|c|}
\hline \multirow[t]{2}{*}{ Element type } & \multirow[t]{2}{*}{$N$} & \multirow[t]{2}{*}{$\|e\|$} & \multicolumn{2}{|l|}{ Low cost } & \multicolumn{2}{|l|}{ Accurate } \\
\hline & & & $\|e\|_{\mathrm{LB}} /\|e\|$ & $\|e\|_{\mathrm{UB}} /\|e\|$ & $\|e\|_{\mathrm{LB}} /\|e\|$ & $\|e\|_{\mathrm{UB}} /\|e\|$ \\
\hline \multirow[t]{7}{*}{ Mini } & 71 & 0.051322 & 0.206753439 & 1.688554616 & 0.244378629 & 1.185885195 \\
\hline & 139 & 0.048175 & 0.139728075 & 1.729050337 & 0 & 1.267503892 \\
\hline & 251 & 0.026226 & 0.136700221 & 1.910699306 & 0.244242355 & 1.246015405 \\
\hline & 499 & 0.022298 & 0.120145305 & 2.106646336 & 0.023359943 & 1.501928424 \\
\hline & 947 & 0.013478 & 0.116627096 & 1.996512836 & 0.270522333 & 1.269105208 \\
\hline & 1891 & 0.010508 & 0.108897982 & 2.261039208 & 0.105376856 & 1.556909022 \\
\hline & 3683 & 0.0067402 & 0.059099137 & 2.130203852 & 0.463220676 & 1.472285689 \\
\hline \multirow[t]{7}{*}{ Taylor-Hood } & 95 & 0.0142 & 0.385957746 & 1.491619718 & 0.523492958 & 1.227394366 \\
\hline & 187 & 0.0076095 & 0.564977988 & 1.142124975 & 0.535370261 & 0.984650765 \\
\hline & 331 & 0.0047997 & 0.375856824 & 1.34837594 & 0.497843615 & 1.1471967 \\
\hline & 659 & 0.0028179 & 0.592391497 & 1.23105859 & 0.692288584 & 1.04854679 \\
\hline & 1235 & 0.0012486 & 0.424115009 & 1.30217844 & 0.50505366 & 1.146403972 \\
\hline & 2467 & 0.00073723 & 0.663117345 & 1.175291293 & 0.706468809 & 1.03524002 \\
\hline & 4771 & 0.00031457 & 0.458498903 & 1.273325492 & 0.507708936 & 1.152970722 \\
\hline \multirow[t]{7}{*}{ P2PO } & 98 & 0.029008 & 0.26698497 & 1.936913955 & - & - \\
\hline & 194 & 0.02226 & 0.289748428 & 2.124887691 & - & - \\
\hline & 354 & 0.015884 & 0.307365903 & 2.101422815 & - & - \\
\hline & 706 & 0.011621 & 0.31855262 & 2.218914035 & - & - \\
\hline & 1346 & 0.0081729 & 0.326616011 & 2.193468659 & - & - \\
\hline & 2690 & 0.0058778 & 0.335873966 & 2.252883732 & - & - \\
\hline & 5250 & 0.0041627 & 0.338145915 & 2.236937565 & - & - \\
\hline \multirow[t]{7}{*}{ Crouxier-Raviart } & 162 & 0.012448 & 0 & 1.120420951 & - & - \\
\hline & 322 & 0.0078446 & 0.022045738 & 1.098258675 & - & - \\
\hline & 610 & 0.0040865 & 0.063000122 & 1.172299033 & - & - \\
\hline & 1218 & 0.0033238 & 0.00623443 & 1.158072086 & - & - \\
\hline & 2370 & 0.0010602 & 0.05701943 & 1.156196944 & - & - \\
\hline & 4738 & 0.00087651 & 0.006702605 & 1.126524512 & - & - \\
\hline & 9346 & 0.00026688 & 0.054934802 & 1.151978417 & - & - \\
\hline
\end{tabular}



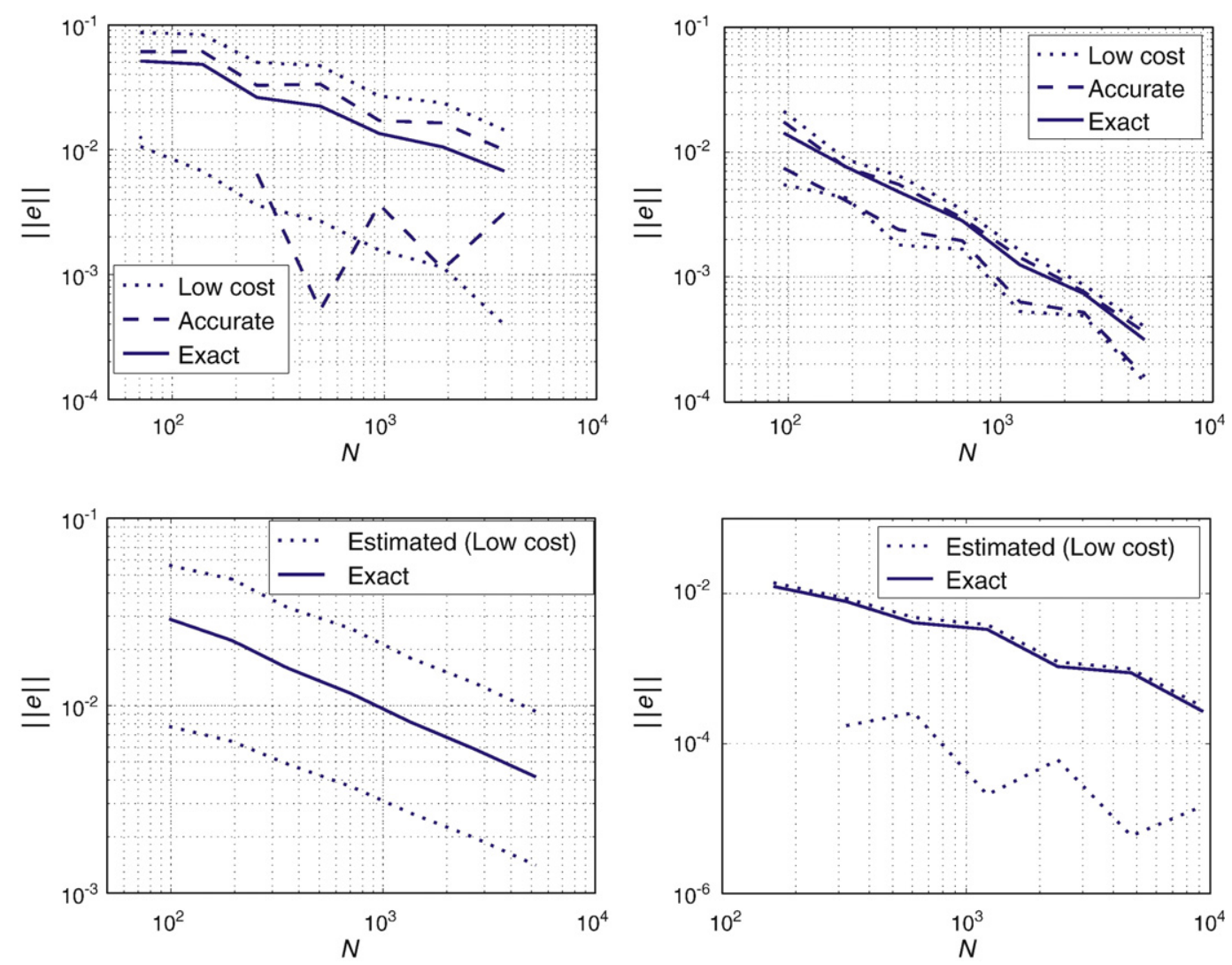

Fig. 3. Convergence of the exact value of and the computed bounds for the velocity-energy norm of the error with increasing number of degrees of freedom for the example of Stokes' flow on the unit square. The approximation was obtained by using Mini-elements (top left), Taylor-Hood elements (top right), P2P0 elements (bottom left) and Crouxier-Raviart elements (bottom right).

denotes the spatial coordinate. The relative bound gap is shown in Fig. 7 for a fixed discretization of 1024 Mini elements using the low cost estimator. As illustrated in the figure, the relative bound gap depends on the chosen goal quantity. Clearly, a more local output is more challenging for a given mesh, and may thus result in a larger relative bound gap. Here, we have only considered radii larger than

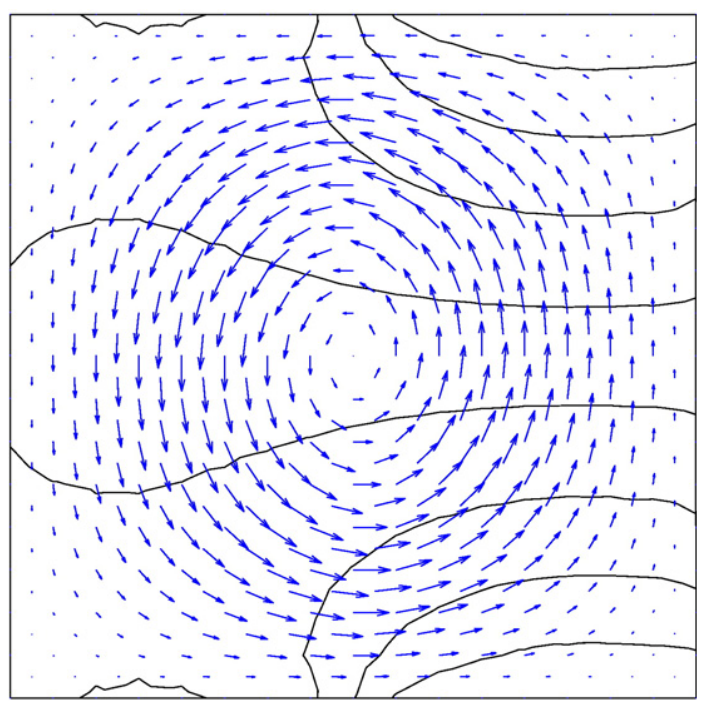

Fig. 4. A numerical solution to the dual problem for the considered output $s$, the averag vertical velocity in the right half, for the example of Stokes' flow on the unit square. Dual velocities are shown together with iso-pressure lines. The approximation was obtained by using Mini-elements and 3683 degrees of freedom. the smallest element in the mesh. For a discussion on measuring even more localized quantities, we refer to, e.g., Prudhomme and Oden [31] or Larsson and Runesson [32].

\subsection{Stokes' channel flow past a step obstacle}

This study aims at investigating the behavior of the presented bounds for more involved problems while elaborating on two output quantities of more local character. The results are limited to the Taylor Hood element and the convergence of the output bounds is presented for uniform mesh refinement. The local problems (of Dirichlet and flux free type) are solved in a manner such that each element $\Omega_{e}$ is subdivided into 25 new elements of the same type.

Following Oden and Prudhomme [7], we study the 2D Stoke's flow in a channel past a single step obstacle, as illustrated in Fig. 8. The modeled part of the channel is of size $40 \times 10$ units and the step is of size $1 \times 1$ and located the distance 5 units from the inlet, as shown in the figure. The inlet (to the left hand side in the figure) is subject to a prescribed parabolic velocity profile with the amplitude 1 unit, whilst the outlet is modeled as traction free. All other boundaries define no slip boundary conditions on the flow. The fluid viscosity is chosen as $\mu=1$. In Fig. 8, a region of interest $A$, which will be used henceforth for defining outputs of interest, is shown. The region is located adjacent to the wall by obstacle on its upstream side and is of size $0.8 \times 0.5$ units.

In Fig. 9, the coarsest and finest mesh used in the study is illustrated. An example of a numerical solution, computed using 1065 degrees of freedom, is illustrated in Fig. 10.

\subsubsection{Estimation of the goal quantity}

We shall now investigate the behavior of the goal oriented error estimators presented in Section 8. To this end, we first define two different outputs of interest for the problem. Firstly, following Oden 
Table 2

Computed relative approximation, bounds and bound gaps for the chosen output quantity $s$ in example of Stokes' flow on a unit square. Results are shown for both the Low cost method and the Accurate method, relying on an additional Dirichlet solution. Using $N$ refers to the total number of degrees of freedom. Note that the exact solution is $s=1 / 240$.

\begin{tabular}{|c|c|c|c|c|c|c|c|c|}
\hline \multirow[t]{2}{*}{ Element type } & \multirow[t]{2}{*}{$N$} & \multirow[t]{2}{*}{$s_{H} / s$} & \multicolumn{3}{|l|}{ Low cost } & \multicolumn{3}{|l|}{ Accurate } \\
\hline & & & $s / s$ & $s^{+} / s$ & $\Delta s / s$ & $s / s$ & $s^{+} / s$ & $\Delta s / s$ \\
\hline \multirow[t]{7}{*}{ Mini } & 71 & 0.297024 & 1.403712 & 1.258608 & 2.66232 & 0.707616 & 1.124664 & 0.417048 \\
\hline & 139 & 0.296376 & 0.0967848 & 1.758792 & 1.8555768 & 0.496128 & 1.726152 & 1.230024 \\
\hline & 251 & 0.797424 & 0.317136 & 1.162416 & 0.84528 & 0.897048 & 1.117776 & 0.220728 \\
\hline & 499 & 0.84576 & 0.637728 & 1.310328 & 0.6726 & 0.923064 & 1.298904 & 0.37584 \\
\hline & 947 & 0.944232 & 0.808296 & 1.055688 & 0.247392 & 0.976224 & 1.040064 & 0.06384 \\
\hline & 1891 & 0.965568 & 0.893448 & 1.071696 & 0.178248 & 0.986952 & 1.06692 & 0.079968 \\
\hline & 3683 & 0.985896 & 0.944808 & 1.020216 & 0.075408 & 0.994368 & 1.014888 & 0.02052 \\
\hline \multirow{7}{*}{ Taylor-Hood } & 95 & 0.978504 & 0.840648 & 1.10784 & 0.267192 & 0.981288 & 1.021344 & 0.040056 \\
\hline & 187 & 0.98112 & 0.983136 & 1.011216 & 0.02808 & 0.991416 & 1.006368 & 0.014952 \\
\hline & 331 & 0.995232 & 0.987168 & 1.009848 & 0.02268 & 0.997272 & 1.0038 & 0.006528 \\
\hline & 659 & 0.997872 & 0.99804 & 1.001328 & 0.003288 & 0.999264 & 1.000656 & 0.001392 \\
\hline & 1235 & 0.999648 & 0.999192 & 1.000632 & 0.00144 & 0.999768 & 1.000272 & 0.000504 \\
\hline & 2467 & 0.99984 & 0.999888 & 1.00008 & 0.000192 & 0.99996 & 1.000032 & $7.2 E \quad 05$ \\
\hline & 4771 & 0.999984 & 0.99996 & 1.000032 & $7.2 E \quad 05$ & 0.999984 & 1.000032 & $4.8 E \quad 05$ \\
\hline \multirow[t]{7}{*}{$P 2 P 0$} & 98 & 0.969456 & 0.356136 & 1.569432 & 1.213296 & - & - & - \\
\hline & 194 & 0.988632 & 0.545448 & 1.409856 & 0.864408 & - & - & - \\
\hline & 354 & 0.99732 & 0.767256 & 1.220448 & 0.453192 & - & - & - \\
\hline & 706 & 0.999024 & 0.862344 & 1.130904 & 0.26856 & - & - & - \\
\hline & 1346 & 0.999816 & 0.933192 & 1.06584 & 0.132648 & - & - & - \\
\hline & 2690 & 0.99996 & 0.963696 & 1.035816 & 0.07212 & - & - & - \\
\hline & 5250 & 0.999984 & 0.982008 & 1.017912 & 0.035904 & - & - & - \\
\hline \multirow[t]{7}{*}{ Crouxier-Raviart } & 162 & 0.955368 & 0.9342 & 1.027656 & 0.093456 & - & - & - \\
\hline & 322 & 0.97932 & 0.971376 & 1.009536 & 0.03816 & - & - & - \\
\hline & 610 & 0.994704 & 0.991224 & 1.003128 & 0.011904 & - & - & - \\
\hline & 1218 & 0.996552 & 0.995064 & 1.001376 & 0.006312 & - & - & - \\
\hline & 2370 & 0.999624 & 0.999312 & 1.000272 & 0.00096 & - & - & - \\
\hline & 4738 & 0.999768 & 0.999648 & 1.000104 & 0.000456 & - & - & - \\
\hline & 9346 & 0.999984 & 0.99996 & 1.000032 & $7.2 E \quad 05$ & - & - & - \\
\hline
\end{tabular}
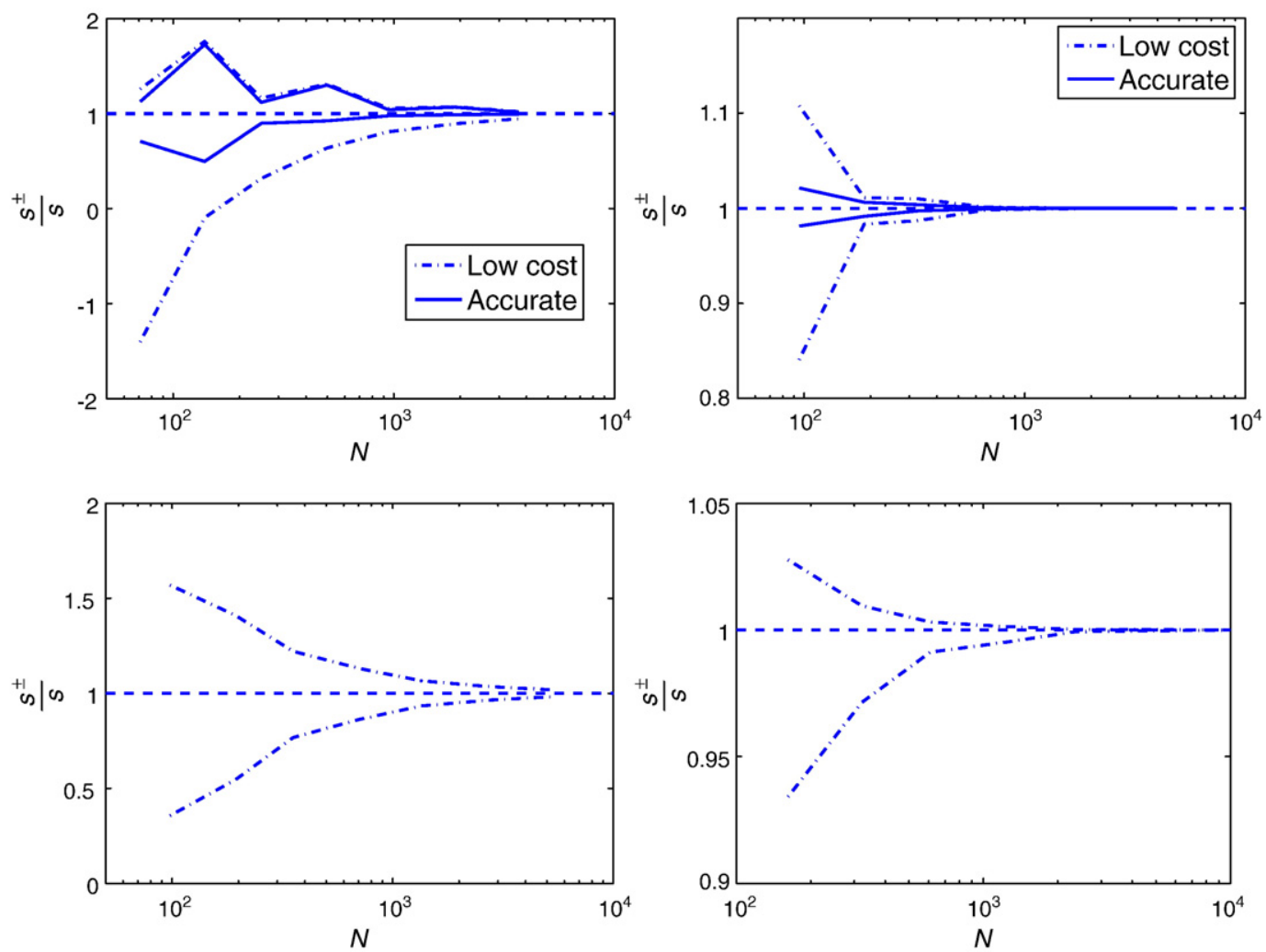

Fig. 5. Computed upper and lower bounds of the output of interest, scaled by the exact value, versus number of degrees of freedom for the example of Stokes' flow on the unit square. The approximation was obtained by using Mini-elements (top left), Taylor-Hood elements (top right), P2P0 elements (bottom left) and Crouxier-Raviart elements (bottom right). 

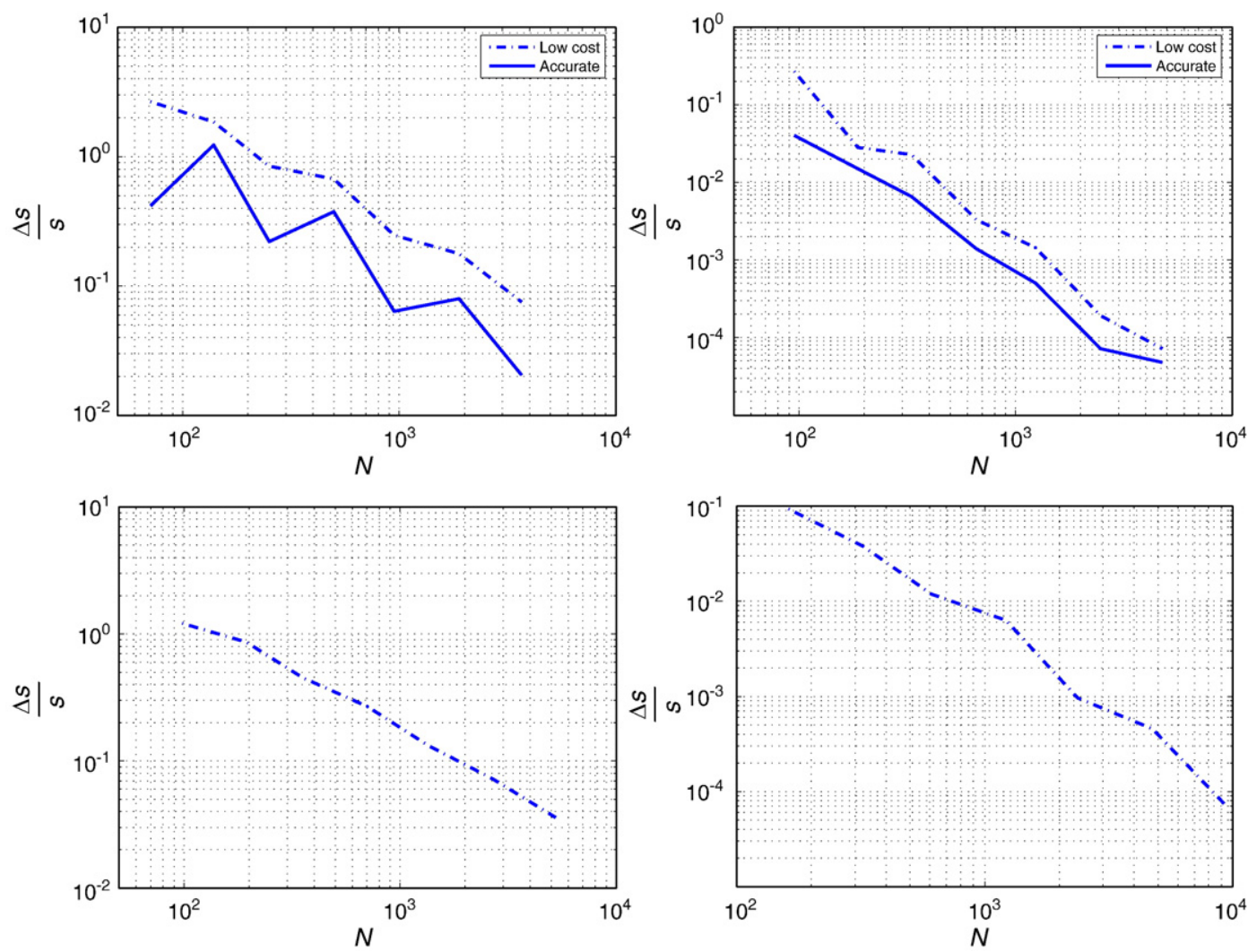

Fig. 6. Convergence of the bound gap, $\Delta s$ with increasing number of degrees of freedom for the example of Stokes' flow on the unit square. The approximation was obtained by using Mini-elements (top left), Taylor-Hood elements (top right), P2P0 elements (bottom left) and Crouxier-Raviart elements (bottom right).
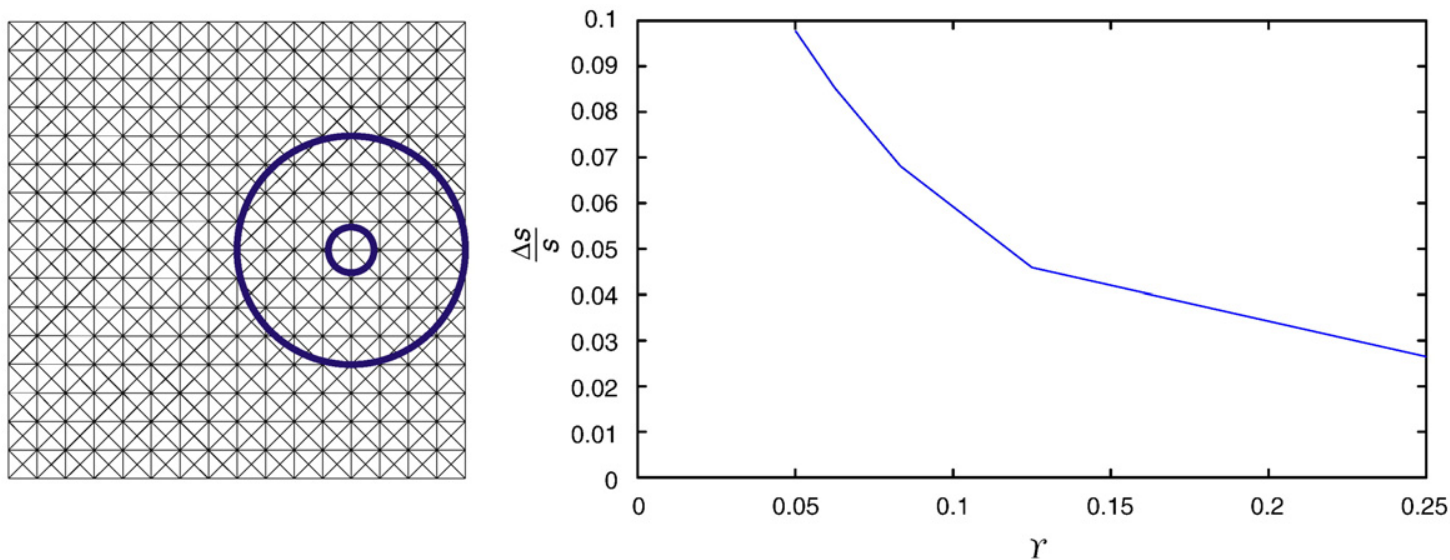

Fig. 7. Relative bound gap versus size of domain for the goal-quantity. Here, the goal-quantity is defined as the average velocity on a circular domain of radius $r$. The approximation was obtained by using a fixed discretization of 1024 Mini-elements, as illustrated in the left figure together with the largest and smallest considered domains.

and Prudhomme [7], we study the circulation around domain $A$, as illustrated in Fig. 8,

$s_{1}=\int_{\partial A} \boldsymbol{u} \cdot \boldsymbol{m} d \Gamma=\int_{\partial A} \boldsymbol{u} \cdot \varepsilon \cdot n d \Gamma$

where $\boldsymbol{m}$ denotes the tangent unit vector and we introduced the transformation tensor ${ }^{1}$

$$
\varepsilon:=-\boldsymbol{e}_{x} \otimes \boldsymbol{e}_{y}+\boldsymbol{e}_{y} \otimes \boldsymbol{e}_{x}
$$

\footnotetext{
${ }^{1}$ We note that the transformation tensor used here in $2 \mathrm{D}$ corresponds to the vector product with the third out of plane direction.
}

where $\boldsymbol{e}_{x}$ and $\boldsymbol{e}_{y}$ denotes the ortho normal unit vectors in $x$ and $y$ direction, respectively. Using the divergence theorem, we may recast the quantity into a domain integral as

$s_{1}=\int_{A} \varepsilon:[\nabla \otimes \boldsymbol{u}]$

which defines our first output quantity of interest. As the second output, we choose to study the average pressure in the same subdomain, i.e.,

$s_{2}=\frac{1}{|A|} \int_{A} p \mathrm{~d} \Omega=l_{p}^{*}(p)$,

where $|A|$ simply denotes the area of the subdomain. 


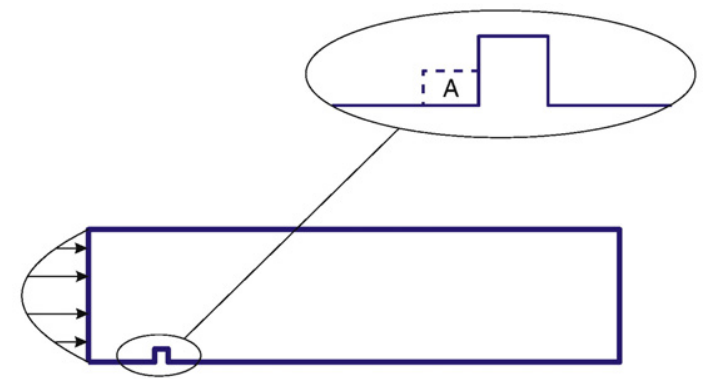

Fig. 8. Illustration of the example problem of Stokes channel flow past a step. The inlet to the left is subject to a prescribed velocity profile whereas the outlet to the right is prescribed to be traction free. The region of interest $A$, defining the outputs of interest.

Examples of the numerical approximations to the pertinent dual problem are shown in Fig. 11. In Table 3, we show the numerical values for the computed upper and lower bounds of the output using both the Low cost bounds, cf. Eq. (198), and the more Accurate bounds using the improved Dirichlet problems, cf. Eq. (208). In Fig. 12, we show the computed upper and lower bounds for chosen goal quantity versus number of degrees of freedom. In Fig. 13, the convergence of the bound gap, $\Delta s$, with mesh refinement is shown. Since the exact solution to this problem is not known, the results in Figs. 12 and 13 are unscaled. We note that, for coarse discretizations, the bound gap is rather large in comparison to the value of the goal quantity. Hence, the outputs studied in this example are more challenging than those studied in Section 9.1. However, the convergence behavior of the bound gap with mesh refinement shows the same, expected, result.

\subsubsection{Computational cost for the procedure}

We now turn to the issue of computational cost. In Fig. 14, the CPU time required for solving the problem is shown versus number of elements in the mesh. The single solution of the FE problem is shown together with the cost of solving the problem and computing the bounds using the low cost and the more accurate bounds, respectively. The cost of computing the bounds is almost entirely governed by the assembly and solution of the local problems. In this example, each element is split into 9 new elements. For reference, we therefore also show the approximate cost of solving the problem on the reference mesh, which is estimated as the cost for solving the global FE problem on a mesh with nine times the number of elements. The academic test example has been solved on a single CPU and without optimizing the practical implementation. Therefore, for coarse meshes, the overhead cost of computing the bounds are very high. In particular, for 115 elements, the cost of computing the bounds are approximately equal to the cost of solving the problem on a reference mesh consisting of $9 \times 115=1035$ elements. However, for finer meshes, we note that the relative overhead cost for computing the bounds reduces drastically. Already for a mesh consisting of 3847 elements, the cost of computing the bounds requires roughly the same amount of CPU time as that of solving the global FE problem itself.

\section{Conclusions}

In this paper we have presented a posteriori error estimation for the Stokes problem. Upper and lower bounds have been derived for the global velocity norm as well as for arbitrary (linear) outputs of interest. The computation of the bounds relies on the solution of local problems on stars or elements. The key feature of the method is the split of the error into two parts; one part capturing the error in the divergence of the solution, which is computable on local Dirichlet problems and the remaining part which can be bounded using local flux free sub domain problems on stars. The presented bounds are guaranteed with respect to a reference solution defined by the mesh density used in the local problems, i.e., they are asymptotic bounds.
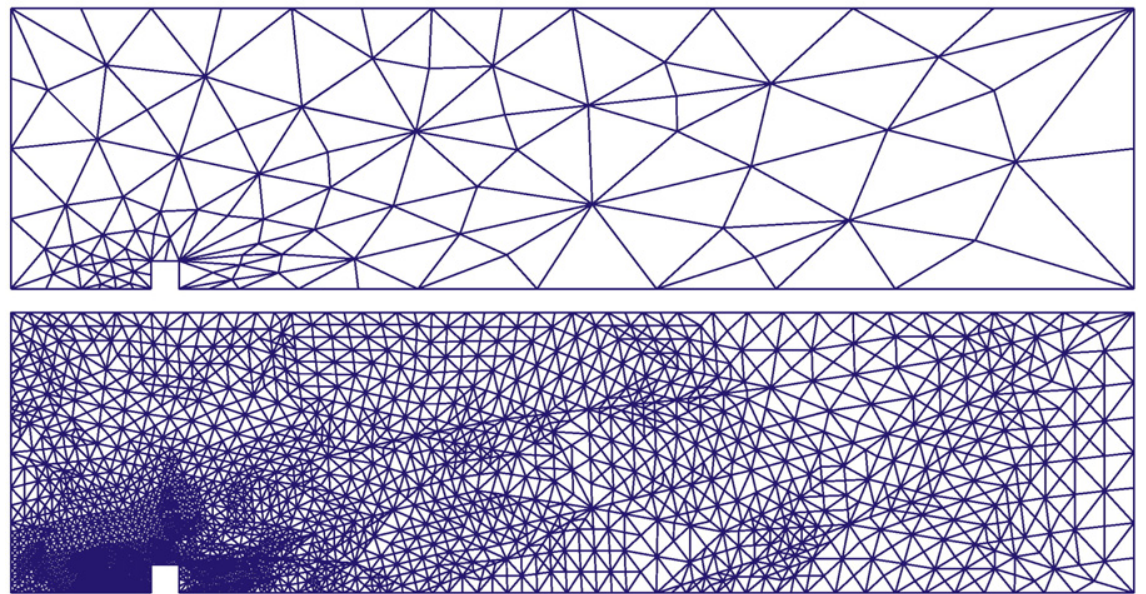

Fig. 9. Coarsest and finest mesh-triangulation used in the example of Stokes' channel flow past a step obstacle. The initial, coarsest, mesh (top) consists of 177 triangular elements, while the most refined mesh (bottom) consists of 5611 elements.

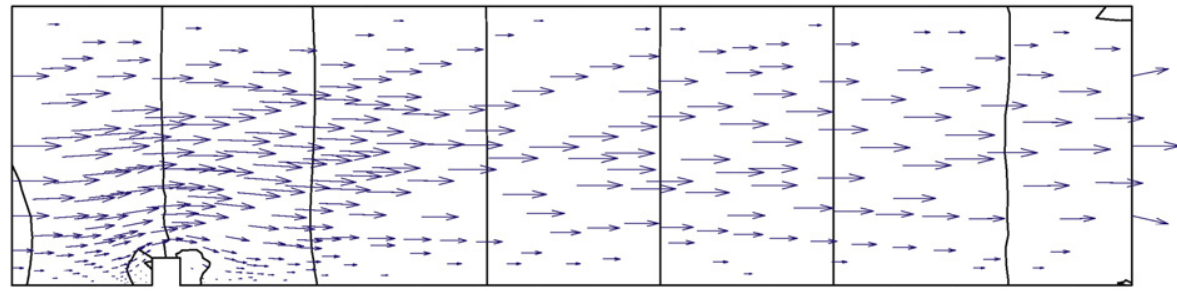

Fig. 10. A numerical solution to the example of Stokes' channel flow past a step obstacle. Velocities are shown together with iso-pressure lines. The approximation was obtained by using Taylor-Hood elements and 5013 degrees of freedom. 

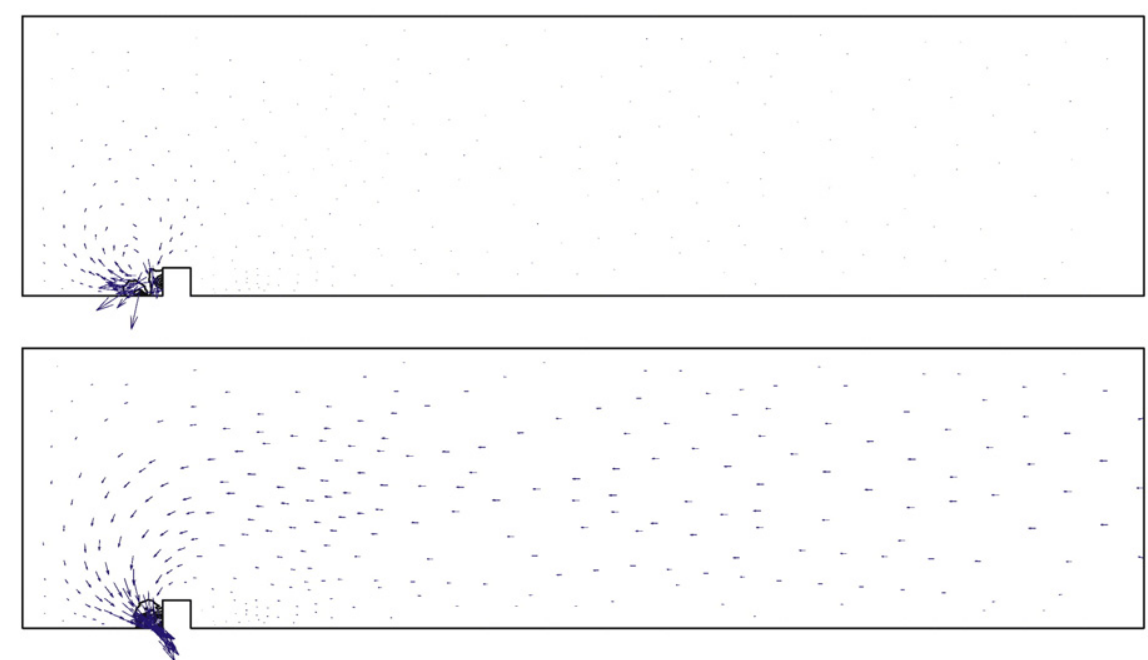

Fig. 11. A numerical solution to the dual problem for the considered outputs $s_{1}$ (top) and $s_{2}$ (bottom) for the example of Stokes' channel flow past a step obstacle. Dual velocities are shown together with iso-pressure lines. The approximation was obtained by using Taylor-Hood elements and 5013 degrees of freedom.

Table 3

Computed relative approximation, bounds and bound gaps for the chosen output quantities in the example of Stokes' channel flow past a step obstacle. Results are shown for both the Low cost method and the Accurate method (relying on an additional Dirichlet solution). $N$ refers to the total number of degrees of freedom for the finite element approximation.

\begin{tabular}{|c|c|c|c|c|c|c|c|c|c|}
\hline Estimator & $N$ & $s_{1, H}$ & $s_{1}$ & $s_{1}^{+}$ & $\Delta s_{1}$ & $s_{2, H}$ & $s_{2}$ & $s_{2}^{+}$ & $\Delta s_{2}$ \\
\hline \multirow[t]{5}{*}{ Low cost } & 897 & 0.0072439 & 0.24965 & 0.063822 & 0.313472 & 3.4839 & 1.8439 & 4.7154 & 2.8715 \\
\hline & 2113 & 0.01338 & 0.070595 & 0.048322 & 0.118917 & 3.4356 & 2.4214 & 4.4465 & 2.0251 \\
\hline & 5013 & 0.016462 & 0.035705 & 0.01166 & 0.047365 & 3.4091 & 3.0411 & 3.8372 & 0.7961 \\
\hline & 11,453 & 0.016414 & 0.027966 & 0.002217 & 0.030183 & 3.4059 & 3.0945 & 3.779 & 0.6845 \\
\hline & 25,760 & 0.0163 & 0.02454 & 0.0051868 & 0.0193532 & 3.4055 & 3.2024 & 3.634 & 0.4316 \\
\hline \multirow[t]{5}{*}{ Accurate } & 897 & 0.0072439 & 0.22301 & 0.029154 & 0.252164 & 3.4839 & 2.4028 & 3.9839 & 1.5811 \\
\hline & 2113 & 0.01338 & 0.049714 & 0.0085784 & 0.0582924 & 3.4356 & 2.8583 & 3.9137 & 1.0554 \\
\hline & 5013 & 0.016462 & 0.026069 & 0.011154 & 0.014915 & 3.4091 & 3.279 & 3.4966 & 0.2176 \\
\hline & 11,453 & 0.016414 & 0.021944 & 0.012182 & 0.009762 & 3.4059 & 3.3103 & 3.4827 & 0.1724 \\
\hline & 25,760 & 0.0163 & 0.01998 & 0.014196 & 0.005784 & 3.4055 & 3.3425 & 3.4503 & 0.1078 \\
\hline
\end{tabular}

Previous existing bounds for outputs of interest for the Stokes problem are restricted to elements with discontinuous pressure approximation, cf. Paraschivoiu, and Patera [30]. The output bounds that have been presented in this paper expand the capabilities of asymptotic bounds to discretization schemes with continuous pressure approximation. Furthermore, pre existing bounds rely on the solution of hybrid fluxes via an equilibration procedure. In this paper, output bounds have been derived based on the solution of sub domain flux free local problems, circumventing the somewhat cumbersome task of solving for the hybrid fluxes.

Two numerical examples have been presented; one case where the exact, analytical solution is known, and one problem from the literature corresponding to an idealized engineering problem. Out puts of interest both in terms of the velocities and the pressure have
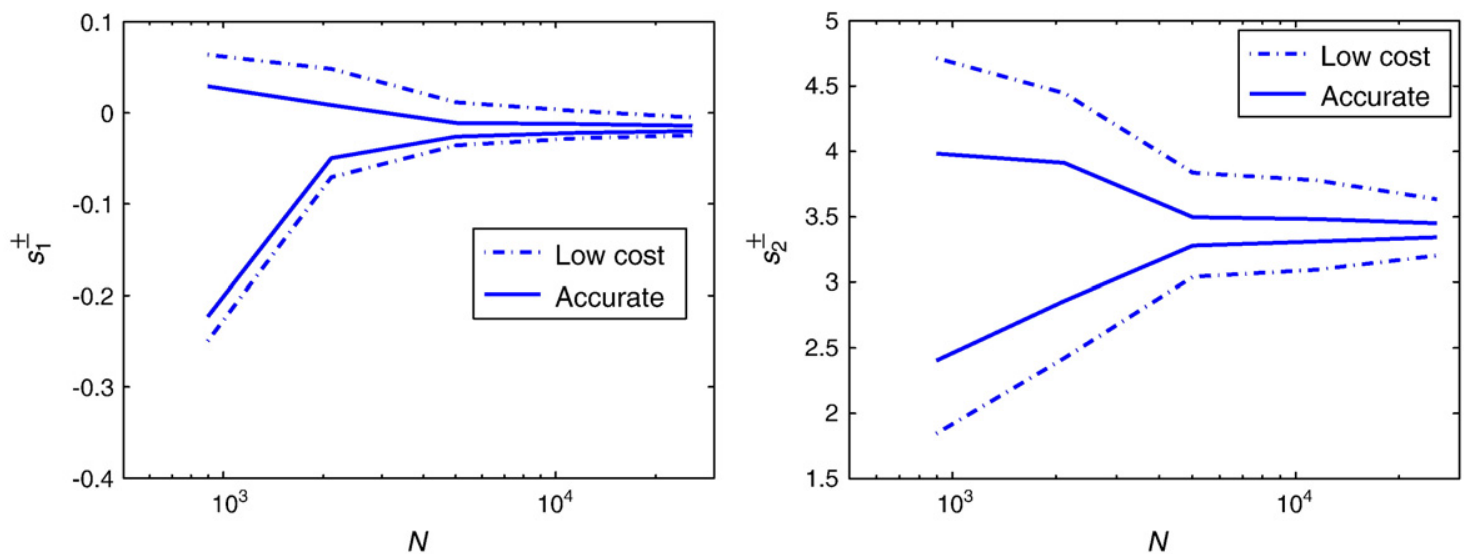

Fig. 12. Computed upper and lower bounds of the outputs of interest, $s_{1}$ (left) and $s_{2}$ (right), versus number of degrees of freedom for the example of Stokes' channel flow past a step obstacle. The approximation was obtained by using Taylor-Hood elements. 

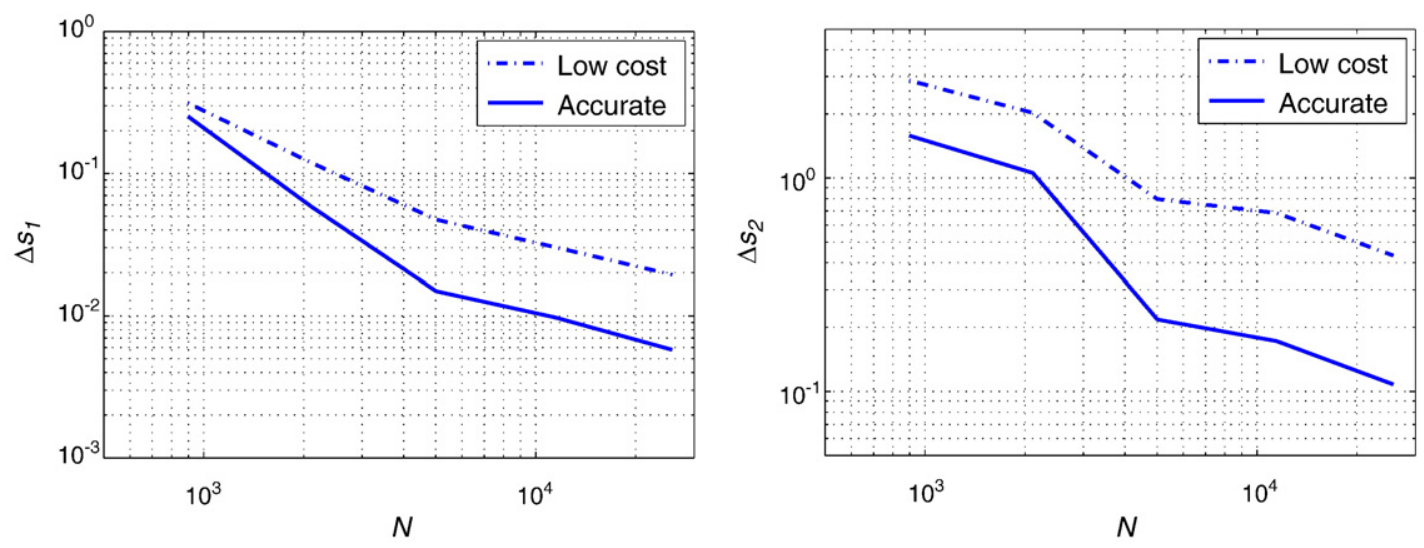

Fig. 13. Convergence of the bound gaps for the two different outputs of interest, $\Delta s_{1}$ (left) and $\Delta s_{2}$ (right), with increasing number of degrees of freedom for the example of Stokes' channel flow past a step obstacle. The approximation was obtained by using Taylor-Hood elements.

been studied. The presented estimators have been shown to be accurate and robust for different element formulations; including both those with continuous and discontinuous pressure approxima tion. In particular, the sharpness of the bounds has been further improved at the extra cost of solving an additional local problem. From the numerical tests, we conclude that the improved bounds are significantly sharper than the ones obtained at the lower cost.

Due to the saddle point nature of the incompressible Stokes problem, at least two local problems are required for each patch, as compared to the single local problem required for an elliptic problem, cf. Pares et al. [20]. Furthermore, we have advocated the solution of a third local problem in order to obtain even sharper bounds. The presented method thus requires some computational effort to produce bounds; however, the computed bounds are robust, accurate and guaranteed with respect to a reference solution defined by the mesh density used in the local problems. Furthermore, we note that the cost of the local problems will increase at a slower rate than the solution of the problem itself.

In this paper, we have presented the a posteriori error analysis. However, the presented output bounds are also well suited for mesh adaptation. The low cost bounds can easily be transformed into refinement indicators following the ideas presented by Pares et al. [20], where the finite element mesh is successively refined in a non

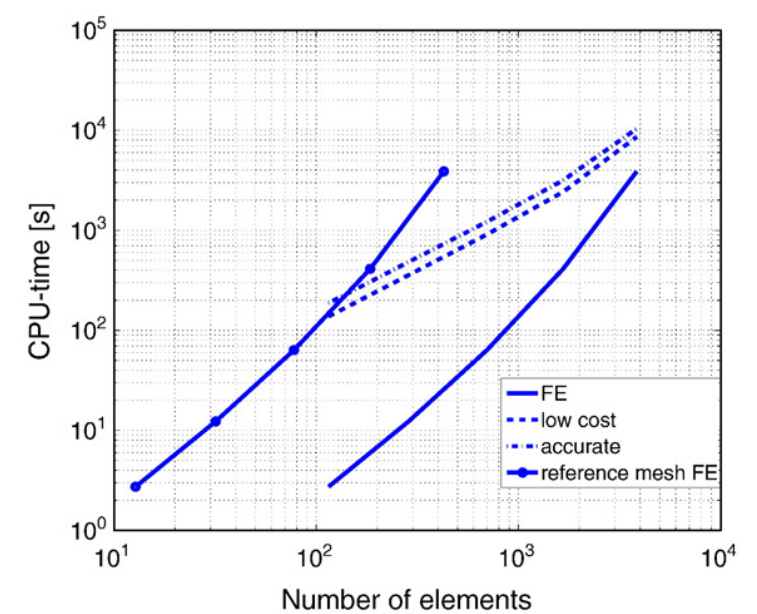

Fig. 14. Computational cost for solving the problem and computing the bounds. The cost of solely computing a single FE-solution (FE) is compared to the cost of solving the problem and computing the bounds on the goal-quantity using the two presented strategies low cost and accurate, respectively. As reference, the computational cost for solving a complete FE-problem on the reference mesh (with 9 times as many elements) is shown. uniform fashion, minimizing the bound gap while using the lowest possible number of degrees of freedom. For the more involved expression obtained for the enhanced (accurate) bounds, the refine ment indicators must be derived using a more general optimization procedure. This is ongoing work left outside the scope of this paper.

The examples studied in this paper are restricted to the use of triangular elements in 2D. The straight forward extension to tetrahe drons in $3 \mathrm{D}$ and more elaborate extensions to quadrilateral and hexagonal elements in 2 and 3 dimensions, respectively, are left outside the scope of this paper. In particular, we stress that the situation that occurs when using hanging nodes needs further analysis.

Finally, we remark that the work presented in this paper is anticipated to serve as a basis for efficient and robust future error estimators for more involved problems such as that of the solving the full Navier Stokes equations, incorporating both convection and non linearities. Furthermore, since the estimator is valid for continuous pressure approximations, it is anticipated that the proposed strategy can be adopted to the coupled problem of deformation of a solid with fluid filled pores.

\section{Acknowledgements}

The authors would like to acknowledge the Graduate School in civil engineering at UPC and the Swedish Research Council (Grant 2003 3697) for the funding of the work.

\section{References}

[1] I. Babuška, C. Rheinboldt, A-posteriori error estimates for the finite element method, Int. J. Numer. Methods Eng. 12 (1978) 1597-1615.

[2] M. Ainsworth, J. Oden, A unified approach to a posteriori error estimation using element residual methods, Numer. Math. 65 (1993) 23-50.

[3] M. Rüter, E. Stein, Analysis, finite element computation and error estimation in transversely isotropic nearly incompressible finite elasticity, Comput. Methods Appl. Mech. Eng. 190 (2000) 519-541.

[4] K. Eriksson, D. Estep, P. Hansbo, C. Johnson, Introduction to adaptive methods for differential equations, Acta Numer. (1995) 105-158.

[5] R. Becker, R. Rannacher, A feed-back approach to error control in finite elemen methods: basic analysis and examples, East-West J. Numer. Math. 4 (1996) 237-264.

[6] W. Bangerth, R. Rannacher, Adaptive Finite Element Methods for Differential Equations, Birkhäuser Verlag AG, 2003.

[7] J. Oden, S. Prudhomme, New approaches to error estimation and adaptivity for the stokes and oseen equations, Int. J. Numer. Meth. Fluids 31 (1999) 3-15.

[8] E. Stein, M. Rüter, S. Ohnimus, Adaptive finite element analysis and modelling of solids and structures. findings, problems and trends, Int. J. Numer. Methods Eng 60 (2004) 103-138.

[9] M. Ainsworth, J. Oden, A Posteriori Error Estimation in Finite Element Analysis, John Wiley \& Sons, New York, 2000.

[10] P. Ladevèze, J.P. Pelle, Mastering calculations in linear and nonlinear mechanics, Mechanical Engineering Series, Springer-Verlag, New York, 2005 (translated from the 2001 French original by Theofanis Strouboulis). 
[11] A. Sauer-Budge, J. Bonet, A. Huerta, J. Peraire, Computing bounds for linear functionals of exact weak solutions to Poisson's equation, SIAM J. Numer. Anal. 42 (4) (2004) 1610-1630 (electronic).

[12] N. Parés, J. Bonet, A. Huerta, J. Peraire, The computation of bounds for linearfunctional outputs of weak solutions to the two-dimensional elasticity equations, Comput. Methods Appl. Mech. Eng. 195 (4-6) (2006) 406-429.

[13] N. Parés, P. Díez, A. Huerta, Bounds of functional outputs for parabolic problems Part I: exact bounds of the discontinuous galerkin time discretization, Comput Methods Appl. Mech. Eng. 197 (19-20) (2008) 1641-1660.

[14] N. Parés, P. Díez, A. Huerta, Bounds of functional outputs for parabolic problems. Part II: bounds of the exact solution, Comput. Methods Appl. Mech. Eng. 197 (19-20) (2008) 1661-1679.

[15] N. Parés, P. Díez, A. Huerta, Exact bounds for linear outputs of the advection-diffusionreaction equation using flux-free error estimates, SIAM J. Sci. Comput. 31 (4) (2009) 3064-3089.

[16] N. Parés, H. Santos, P. Díez, Guaranteed energy error bounds for the Poisson equation using a flux-free approach: solving the local problems in subdomains, Int. J. Numer. Methods Eng. 79 (10) (2009) 1203-1244.

[17] Z.C. Xuan, N. Parés, J. Peraire, Computing upper and lower bounds for the $J$-integral in two-dimensional linear elasticity, Comput. Methods Appl. Mech. Eng. 195 (4-6) (2006) 430-443.

[18] P. Ladevèze, Strict upper error bounds on computed outputs of interest in computational structural mechanics, Comput. Mech. 42 (2) (2008) 271-286.

[19] A. Sauer-Budge, J. Peraire, Computing bounds for linear functionals of exact weak solutions to the advection-diffusion-reaction equation, SIAM J. Sci. Comput. 26 (2004) 636-652.

[20] N. Parés, P. Díez, A. Huerta, Subdomain-based flux-free a posteriori error estimators, Comput. Methods Appl. Mech. Eng. 195 (2006) 297-323.

[21] F. Larsson, P. Hansbo, K. Runesson, Strategies for computing goal-oriented posteriori error measures in non-linear elasticity, Int. J. Numer. Meth. Eng. 55 (2002) 879-894
22] P. Díez, G. Calderón, Remeshing criteria and proper error representations for goal oriented h-adaptivity, Comput. Methods Appl. Mech. Eng. 196 (2007) 719-733.

[23] W. Dorfler, A convergent adaptive algorithm for Poisson's equation, SIAM J. Numer. Anal. 33 (3) (1996) 1106-1124.

[24] P. Morin, R. Nochetto, K. Siebert, Data oscillation and convergence of adaptive FEM, SIAM J. Numer. Anal. 38 (2) (2000) 466-488.

[25] K. Moon, A. Szepessy, R. Tempone, G. Zouraris, Convergence rates for adaptive approximation of ordinary differential equations, Numer. Math. 96 (1) (2003) 99-129.

[26] P. Binev, W. Dahmen, R. DeVore, Adaptive finite element methods with convergence rates, Numer. Math. 97 (2) (2004) 219-268.

[27] J. Donea, A. Huerta, Finite Element Methods for Flow Problems, John Wiley \& Sons Ltd., Chichester, 2003.

28] H. Melbø, T. Kvamsdal, Goal oriented error estimators for stokes equations based on variationally consistent postprocessing, Comput. Methods Appl. Mech. Eng. 192 (2003) 613-633.

[29] L. Machiels, J. Peraire, A. Patera, A posteriori finite-element output bounds for the incompressible Navier-Stokes equations: application to a natural convection problem, J. Comput. Phys. 172 (2001) 401-425.

[30] M. Paraschivoiu, A. Patera, A posteriori bounds for linear functional outputs of Crouzeix-Raviart finite element discretizations of the incompressible stokes problem, Int. J. Numer. Meth. Fluids 32 (2000) 823-849.

[31] S. Prudhomme, J.T. Oden, On goal-oriented error estimation for elliptic problems: application to the control of pointwise errors, Comput. Methods Appl. Mech. Eng. 176 (1999) 313-331.

[32] F. Larsson, K. Runesson, Rve computations with error control and adaptivity: the power of duality, Comput. Mech. 39 (2007) 647-661. 